\title{
Multi-color observations of short GRB afterglows: 20 events observed between 2007 and 2010*
}

\author{
A. Nicuesa Guelbenzu ${ }^{1}$, S. Klose ${ }^{1}$, J. Greiner ${ }^{2}$, D. A. Kann ${ }^{1}$, T. Krühler ${ }^{3}$, A. Rossi ${ }^{1}$, S. Schulze ${ }^{4}$, P. M. J. Afonso ${ }^{5}$, \\ J. Elliott ${ }^{2}$, R. Filgas ${ }^{2,6}$, D. H. Hartmann ${ }^{7}$, A. Küpcü Yoldaş ${ }^{8}$, S. McBreen ${ }^{9}$, M. Nardini ${ }^{10}$, F. Olivares E. ${ }^{2}$, A. Rau ${ }^{2}$, \\ S. Schmidl ${ }^{1}$, P. Schady ${ }^{2}$, V. Sudilovsky ${ }^{2}$, A. C. Updike ${ }^{11}$, and A. Yoldaş ${ }^{8}$
}

1 Thüringer Landessternwarte Tautenburg, Sternwarte 5, 07778 Tautenburg, Germany e-mail: ana@tls-tautenburg.de

2 Max-Planck-Institut für Extraterrestrische Physik, Giessenbachstraße, 85748 Garching, Germany

3 Dark Cosmology Centre, Niels Bohr Institute, University of Copenhagen, Juliane Maries Vej 30, 2100 Copenhagen, Denmark

4 Centre for Astrophysics and Cosmology, Science Institute, University of Iceland, Dunhagi 5, 107 Reykjavík, Iceland

5 American River College, Department of Physics and Astronomy, 4700 College Oak Drive, Sacramento, CA 95841, USA

6 Institute of Experimental and Applied Physics, Czech Technical University in Prague, Horská 3a/22, 12800 Prague 2, Czech Republic

7 Clemson University, Department of Physics and Astronomy, Clemson, SC 29634-0978, USA

8 Institute of Astronomy, University of Cambridge, Madingley Road CB3 OHA, Cambridge, UK

9 School of Physics, University College Dublin, Dublin 4, Republic of Ireland

10 Università degli studi di Milano-Bicocca, Piazza della Scienza 3, 20126 Milano, Italy

11 Department of Astronomy, University of Maryland, College Park, MD 20742, USA

Received 4 May 2012 / Accepted 25 July 2012

\section{ABSTRACT}

\begin{abstract}
We report on follow-up observations of 20 short-duration gamma-ray bursts (GRBs; $T_{90}<2$ s) performed in $g^{\prime} r^{\prime} i^{\prime} z^{\prime} J H K_{\mathrm{s}}$ with the Gamma-Ray Burst Optical Near-Infrared Detector (GROND) between mid-2007 and the end of 2010. This is one of the most comprehensive data sets on GRB afterglow observations of short bursts published so far. In three cases, GROND was on target within less than 10 min after the trigger, leading to the discovery of the afterglow of GRB 081226A and its faint underlying host galaxy. In addition, GROND was able to image the optical afterglow and follow the light curve evolution in five further cases: GRBs 090305, 090426, 090510, 090927, and 100117A. In all other cases, optical/near-infrared upper limits can be provided on the afterglow magnitudes. After shifting all light curves to a common redshift, we find that the optical luminosities of the six events with light curves group into two subsamples. GRBs 090426 and 090927 are situated in the regime occupied by long-duration events (collapsars), while the other four bursts occupy the parameter space typical for merger events, confirming that the short-burst population is contaminated by collapsar events. Three of the aforementioned six bursts with optical light curves show a break: GRBs 090426 and 090510 (Papers I and II) as well as GRB 090305. For GRB 090927, no break is seen in the optical/X-ray light curve until about $150 \mathrm{ks} / 600 \mathrm{ks}$ after the burst. The GROND multi-color data support the view that this burst is related to a collapsar event. A decay slope of the optical afterglow of GRB 100117A could be measured. For all six GRBs a lower limit on the corresponding jet opening angle can be set. Using these data supplemented by about ten events taken from the literature, we compare the jet half-opening angles of long and short bursts. We find tentative evidence that short bursts have wider opening angles than long bursts. However, the statistics are still very poor.
\end{abstract}

Key words. gamma-ray burst: general

\section{Introduction}

Gamma-ray bursts (GRBs) show a bimodality in their duration distribution, separated in the Compton GammaRay Observatory/Burst And Transient Source Experiment (CGRO/BATSE) data at $T_{90}=2 \mathrm{~s}$, with the peak of the shortburst population at $T_{90} \sim 0.5 \mathrm{~s}$ and the long-burst population at $\sim 30 \mathrm{~s}$ (Kouveliotou et al. 1993; Sakamoto et al. 2011). Historically, bursts are divided into long and short based on the BATSE scheme, even though the shape of the bimodal distribution is energy-dependent, in particular peaking for Swift/Burst Alert Telescope (BAT) at $T_{90} \sim 0.5 \mathrm{~s}$ and $\sim 70 \mathrm{~s}$, respectively (Sakamoto et al. 2011).

According to the current picture, long bursts originate from the collapse of massive stars into black holes (MacFadyen \& Woosley 1999) or into rapidly spinning, strongly magnetized

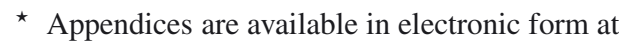
http://www . aanda.org neutron stars (e.g., Usov 1992; Mazzali et al. 2006). Short bursts, on the other hand, are commonly attributed to the merger of compact stellar objects (e.g., Paczyński 1986; Nakar 2007). The physical association of long bursts with the collapse of massive stars has been well established (e.g., Zeh et al. 2004; Hjorth et al. 2003; Kawabata et al. 2003; Matheson et al. 2003; Stanek et al. 2003; Pian et al. 2006; Ferrero et al. 2006; Woosley \& Bloom 2006; Fruchter et al. 2006). However, the observational situation with short bursts is less clear.

Until 2005 no afterglow of a short burst had ever been detected, while many important discoveries had already been made for the long-burst sample (redshifts, supernova light, collimated explosions, circumburst wind profiles). The first well-localized short burst (GRB 050509B; Gehrels et al. 2005) was seen close in projection to a massive early-type galaxy (Hjorth et al. 2005; Bloom et al. 2006), supporting the model that compact stellar mergers are the progenitors of short-duration gamma-ray bursts. However, since then observational progress has been 
rather modest compared to the long-burst population (for recent reviews, see Gehrels et al. 2009; Berger 2011).

There are two main reasons for this situation. Firstly, there is a substantially smaller detection rate of short bursts compared to long bursts. Secondly, short-burst afterglows are rarely brighter than $R=20$, even minutes after a trigger (e.g., Kann et al. 2010, 2011). This general faintness makes their discovery and detailed follow-up very challenging. However, only the precise detection of the afterglow, with sub-arcsec accuracy, enables a secure determination of a putative GRB host galaxy and its redshift, while the X-ray plus optical light curves provide information about the processes after the explosion and the properties of the environment of the progenitor along with clues about the physics of the central engine. Rapid follow-up observations of these events are therefore very important to gain as much observational data as possible.

Since there is a substantial overlap between the long- and short-burst duration distribution, a simple division into long and short is only a first guess about the true origin of a burst. Several other phenomenological properties of the bursts and their afterglows have to be considered in order to reveal the nature of their progenitors (Zhang et al. 2007, 2009; Kann et al. 2011). Particularly interesting are the circumburst density profiles, the afterglow luminosities, and the outflow characteristics that might be shaped by or related to the physical properties of the GRB progenitors.

Theoretical studies suggest that long GRBs are followed by more luminous afterglows than short bursts, mainly due to the expected difference in the circumburst density, around the GRB progenitors (Panaitescu et al. 2001). In addition, the circumburst density profile indicates the nature of the explosion (e.g., Schulze et al. 2011). The distribution functions of the jetopening angles of long and short bursts should also be different from each other because an extended massive envelope collimates the escaping relativistic outflow (Zhang et al. 2004), while the lack of such a medium in merger events might allow for wider jet-opening angles (Aloy et al. 2005; Rezzolla et al. 2011). Any short-burst afterglow that adds information here is of great interest.

Here we report on the results of the first 3.5 years of followup observations of short-duration GRBs using the optical/nearinfrared (NIR) seven-channel imager GROND (Greiner et al. 2007, 2008) mounted at the 2.2-m ESO/MPG telescope on La Silla (Chile). GROND has been in continuous operation since mid-2007, observing every burst with a declination $\lesssim+35^{\circ}$. It thus provides a complete sample of events observed with the same instrument at the same telescope. The capability of GROND to observe in seven bands simultaneously, from $g^{\prime}$ to $K_{\mathrm{s}}$, not only provides the opportunity to follow the color evolution of an afterglow but also allows for a stacking of all bands. This is particularly the case for a white-light image in $g^{\prime} r^{\prime} i^{\prime} z^{\prime}$, which reaches a fainter detection threshold. In addition, GROND's routine operation in rapid response mode allows us to start observations within minutes after a trigger, thereby catching afterglows even if they are fading rapidly.

In this work, we summarize the detections and upper limits for 20 short-burst afterglows in $g^{\prime} r^{\prime} i^{\prime} z^{\prime} J H K_{\mathrm{s}}$. First results have already been published in Nicuesa Guelbenzu et al. (2011, in the following Paper I) and Nicuesa Guelbenzu et al. (2012, in the following Paper II). Here we add detailed information on all individual bursts. In particular, we compare the afterglow luminosities with those of their long-burst relatives. We also include X-ray data in order to extend this discussion to the highenergy band. If possible, based on our optical data, we derive the spectral energy distribution (SED) of the afterglows and give an estimate of the corresponding jet half-opening angles.

In the following, we mainly are in line with the classical classification scheme into long and short bursts with the border line at $T_{90}=2 \mathrm{~s}$ (Kouveliotou et al. 1993). We stress, however, that this is a matter of discussion and a matter of detector properties (Sakamoto et al. 2011), and a more physically motivated classification scheme is often used (Zhang et al. 2007; Kann et al. 2010, 2011). This revised scheme was basically triggered by GRBs 060605 and 060614. Despite a low redshift, these two long bursts in the classical sense developed no detectable supernova component down to deep luminosity limits (Della Valle et al. 2006; Fynbo et al. 2006; Gal-Yam et al. 2006; Gehrels et al. 2006; Thöne et al. 2008).

Throughout the paper, we adopt a concordance $\Lambda$ CDM cosmology $\left(\Omega_{\mathrm{M}}=0.27, \Omega_{\Lambda}=0.73, H_{0}=71 \mathrm{~km} \mathrm{~s}^{-1} \mathrm{Mpc}^{-1}\right.$; Spergel et al. 2003) and describe the flux density as $F_{v}(t) \propto$ $t^{-\alpha} v^{-\beta}$. In cases where no redshift is known for a burst, we adopt a redshift of $z=0.5$, which is justified based on the redshift distribution of short bursts detected by Swift by the end of 2010 (Leibler \& Berger 2010, their Table 1).

\section{Target selection, observations, and data reduction}

Between July 2007 and December 2010, a total of 394 GRBs were localized at the arcmin or (mostly) arcsec scale ${ }^{1}$. Among these 220 events were followed up with GROND. For the present study, we have selected from this data base all those bursts with a duration of $T_{90} \leq 2 \mathrm{~s}$ (within $1 \sigma$ ) and an error circle smaller than 3 arcmin in radius (Table 1), giving us 20 targets.

All optical/NIR data were analysed through standard point spread function (PSF) photometry using DAOPHOT and ALLSTAR tasks of IRAF (Tody 1993) in a similar way to the procedure described in Krühler et al. (2008) and Küpcü Yoldaş et al. (2008). A PSF fitting was used to measure the magnitudes of an optical transient. For completeness, publicly available archives were also checked (Very Large Telescope (VLT) with the instrument Focal Reducer and low dispersion Spectrograph (FORS) and Gemini with the Gemini Multi-Object Spectrographs (GMOS)).

The optical data were calibrated against the Sloan Digital Sky Survey (SDSS DR7; Abazajian et al. 2009), if available. Otherwise a standard star field was observed under photometric conditions. For the NIR bands, photometric calibration was performed against the Two Micron All Sky Survey (2MASS) catalogue (Skrutskie et al. 2006). This procedure results in a typical absolute accuracy of $0.04 \mathrm{mag}$ in $g^{\prime} r^{\prime} i^{\prime} z^{\prime}, 0.06 \mathrm{mag}$ in $J H$, and 0.08 mag in $K_{\mathrm{s}}$. All reported magnitudes are in the $\mathrm{AB}$ photometric system. Observed magnitudes were corrected for Galactic reddening based on Schlegel et al. (1998) and assuming a Milky Way extinction curve with a ratio of total-toselective extinction of $R_{V}=3.1$. For GROND, the Vega-to-AB conversion is $J_{\mathrm{AB}}=J_{\text {Vega }}+0.93 \mathrm{mag}, H_{\mathrm{AB}}=H_{\mathrm{Vega}}+1.39 \mathrm{mag}$, $K_{\mathrm{AB}}=K_{\mathrm{s} \text {, Vega }}+1.80 \mathrm{mag}$, except for observations after an intervention on the instrument on March 2008, for which $K_{\mathrm{AB}}=$ $K_{\mathrm{s}, \text { Vega }}+1.86 \mathrm{mag}$. Extinction corrections for the GROND filters we used here are $A\left(g^{\prime}\right)=1.253 A_{V}, A\left(r^{\prime}\right)=0.799 A_{V}, A\left(i^{\prime}\right)=$ $0.615 A_{V}, \quad A\left(z^{\prime}\right)=0.454 A_{V}, \quad A(J)=0.292 A_{V}, \quad A(H)=$ $0.184 A_{V}, A\left(K_{\mathrm{s}}\right)=0.136 A_{V}$.

http://www.mpe.mpg.de/ jcg/grbgen.html 
Table 1. The 20 short bursts of our sample.

\begin{tabular}{lccccccccccc}
\hline \hline$\#$ & GRB & RA (J2000) & $\begin{array}{c}\text { Dec } \\
(4)\end{array}$ & $\begin{array}{c}\text { Inst. } \\
(5)\end{array}$ & $\begin{array}{c}\text { Error }\left[{ }^{\prime \prime}\right] \\
(6)\end{array}$ & $\begin{array}{c}\text { Ref. } \\
(7)\end{array}$ & $\begin{array}{c}T_{90}[\mathrm{~s}] \\
(8)\end{array}$ & $\begin{array}{c}\text { Ref. } \\
(9)\end{array}$ & $\begin{array}{c}E(B-V) \\
(10)\end{array}$ & $\begin{array}{c}z \\
(11)\end{array}$ & $\begin{array}{c}\text { Ref. } \\
(12)\end{array}$ \\
\hline 1 & 070729 & $03: 45: 16.02$ & $-39: 19: 20.6$ & XRT & 2.5 & 1 & $0.9 \pm 0.1$ & 1 & 0.02 & - & - \\
2 & $071112 B$ & $17: 20: 51.0$ & $-80: 53: 02$ & BAT & 132 & 2 & $0.3 \pm 0.05$ & 2 & 0.12 & - & - \\
3 & 071227 & $03: 52: 31.26$ & $-55: 59: 03.5$ & OT & 0.3 & 4 & $1.8 \pm 0.4$ & 3 & 0.01 & 0.381 & 39 \\
4 & $080905 A$ & $19: 10: 41.73$ & $-18: 52: 47.3$ & OT & 0.6 & 6,7 & $1.0 \pm 0.1$ & 5 & 0.14 & 0.122 & 41 \\
5 & 080919 & $17: 40: 53.78$ & $-42: 22: 05.7$ & XRT & 1.6 & 8 & $0.6 \pm 0.1$ & 8 & 0.49 & - & - \\
6 & $081226 \mathrm{~A}$ & $08: 02: 00.45$ & $-69: 01: 49.5$ & OT & 0.2 & this work & $0.4 \pm 0.1$ & 9 & 0.16 & - & - \\
7 & $081226 \mathrm{~B}$ & $01: 41: 59$ & $-47: 26: 19$ & IBIS & 150 & 11 & 0.7 & 11 & 0.02 & - & - \\
8 & 090305 & $16: 07: 07.59$ & $-31: 33: 21.9$ & OT & 0.2 & this work & $0.4 \pm 0.1$ & 14 & 0.22 & - & - \\
9 & 090426 & $12: 36: 18.07$ & $+32: 59: 09.6$ & OT & 0.5 & this work & $1.2 \pm 0.3$ & 17 & 0.02 & 2.609 & 32 \\
10 & 090510 & $22: 14: 12.50$ & $-26: 34: 59.0$ & OT & 0.2 & 42 & $0.3 \pm 0.1$ & 18 & 0.02 & 0.903 & 33,43 \\
11 & 090927 & $22: 55: 53.39$ & $-70: 58: 49.50$ & OT & 0.2 & this work & $2.2 \pm 0.4$ & 19 & 0.03 & 1.37 & 34 \\
12 & $091109 B$ & $07: 30: 56.61$ & $-54: 05: 22.85$ & OT & 0.5 & 20,38 & $0.3 \pm 0.03$ & 21 & 0.03 & - & - \\
13 & $091117 A$ & $02: 03: 46.9$ & $-16: 56: 38$ & BAT & 156 & 22,23 & $0.43 \pm 0.05$ & 24 & 0.03 & - & - \\
14 & $100117 A$ & $00: 45: 04.66$ & $-01: 35: 41.89$ & OT & 0.26 & 40 & $0.30 \pm 0.05$ & 25 & 0.02 & 0.915 & 40 \\
15 & $100206 \mathrm{~A}$ & $03: 08: 39.03$ & $+13: 09: 25.3$ & XRT & 3.3 & 26 & $0.12 \pm 0.03$ & 26 & 0.38 & 0.41 & 35 \\
16 & $100625 \mathrm{~A}$ & $01: 03: 10.91$ & $-39: 05: 18.4$ & XRT & 1.8 & 27 & $0.33 \pm 0.03$ & 27 & 0.01 & - & - \\
17 & $100628 \mathrm{~A}$ & $15: 03: 52.41$ & $-31: 39: 30.2$ & XRT & 7.0 & 28 & $0.036 \pm 0.009$ & 28 & 0.17 & 0.102 & 36 \\
18 & $100702 \mathrm{~A}$ & $16: 22: 47.26$ & $-56: 31: 53.8$ & XRT & 2.4 & 29 & $0.16 \pm 0.03$ & 29 & 0.41 & - & - \\
19 & $101129 \mathrm{~A}$ & $10: 23: 41$ & $-17: 38: 42$ & BAT & 180 & 30 & $0.35 \pm 0.05$ & 30 & 0.07 & - & - \\
20 & $101219 \mathrm{~A}$ & $04: 58: 20.49$ & $-02: 32: 23.0$ & XRT & 1.7 & 31 & $0.6 \pm 0.2$ & 32 & 0.06 & 0.718 & 37 \\
\hline
\end{tabular}

Notes. Columns 5-7 give the instrument on which the coordinates are based (OT stands for optical transient detected), the corresponding radius of the error circle, and the reference, respectively. BAT and XRT stand for the instruments onboard of the Swift satellite, IBIS stands for the instrument onboard the INTEGRAL satellite. Columns 8 and 9 provide $T_{90}$ and the corresponding reference. The last columns give the Galactic reddening $E(B-V)$ (mag) along the line of sight according to Schlegel et al. (1998) as well as the redshift. If available, enhanced Swift/XRT positions are given in Cols. 3 and 4 as well as the revised error circles, taken from http://www.swift.ac.uk/xrt_positions/index.php and Evans (2011a,b).

References. 1 = Guidorzi et al. (2007c); 2 = Perri et al. (2007); 3 = Sakamoto et al. (2007b); 4 = D' Avanzo et al. (2008); $5=$ Pagani et al. (2008b); $6=$ Malesani et al. (2008); $7=$ de Ugarte Postigo et al. (2008); $8=$ Preger et al. (2008b); $9=$ Krimm et al. (2008); 11= Mereghetti et al. (2008); $14=$ Krimm et al. (2009); $17=$ Sato et al. (2009); $18=$ Hoversten et al. (2009); $19=$ Grupe et al. (2009b); $20=$ Levan et al. $(2009 \mathrm{~b}) ; 21=$ Oates et al. (2009b); 22 = Cummings et al. (2009); 23 = D'Elia et al. (2009); 24 = Sakamoto et al. (2009); $25=$ de Pasquale et al. (2010c); $26=$ Krimm et al. (2010c); 27 = Holland et al. (2010b); $28=$ Immler et al. (2010); $29=$ Siegel et al. (2010b); $30=$ Cummings et al. (2010); $31=$ Gelbord et al. (2010); $32=$ Krimm et al. (2010a); $32=$ Levesque et al. (2009); $33=$ Rau et al. (2009); $34=$ Levan et al. (2009a); $35=$ Cenko et al. (2010a); $36=$ Cenko et al. (2010b); 37 = Chornock \& Berger (2011); $38=$ Malesani et al. (2009); $39=$ D' Avanzo et al. (2009); $40=$ Fong et al. (2011); $41=$ Rowlinson et al. (2010); $42=$ Nicuesa Guelbenzu et al. (2012); $43=$ McBreen et al. (2010).

\section{Results}

In the following, we combined GROND's $g^{\prime} r^{\prime} i^{\prime} z^{\prime}$ into a white band in several cases. This turned out to be particularly useful when searching for a faint afterglow, studying the light curve shape, and measuring the offset of a detected afterglow from its suspected host galaxy. Image subtraction between the first and the last epoch was performed using the hotpants package ${ }^{2}$. Errors in the astrometric accuracy of GROND are less than 0.'3 in right ascension and declination.

\subsection{GRBs with an afterglow detected by GROND}

In six of the 20 events followed up by GROND, an optical afterglow was detected by GROND. Two of these events, GRB 090426 and GRB 090510, were discussed in detail in Papers I and II. Here we report on the four other cases.

\footnotetext{
2 http://www.astro.washington.edu/users/becker/ hotpants.html

http://svn.pan-starrs.ifa.hawaii.edu/trac/ipp/wiki/ ppSub_vs_Hotpants
}

\subsubsection{GRB 081226A: discovery of the optical afterglow}

Observations: GROND started observations $10 \mathrm{~min}$ after the GRB trigger and was on target for $2.5 \mathrm{~h}$. Second-epoch observations were performed the following night, and a final epoch was obtained one month after the burst. Inside the $90 \%$ c.l. X-ray Telescope (XRT) error circle $\left(r=33^{\prime \prime} 8\right.$; Evans 2011a,b), the white-band image shows three objects (A-C; Fig. 1).

Afterglow light curve: after image subtraction is performed on the white-band images, the afterglow appears in the southern part of its very faint host galaxy (object $\mathrm{C}$ in Fig. 1). It is detected in all optical bands (Table A.1) and is best-sampled in the $r^{\prime}$ band. Fitting the light curve with a single power-law plus host galaxy component (Fig. 2) gives $\alpha=1.3 \pm 0.2$ (i.e., the afterglow was in the pre-jet break evolutionary phase). The decay slope is in agreement with the two X-ray detections of the afterglow centered at $0.6 \mathrm{ks}$ and $11.5 \mathrm{ks}$ (Evans et al. 2010). We re-reduced archival Gemini $r^{\prime}$-band images (Berger et al. 2008a) and found that they fit well into this light curve, confirming the GROND discovery.

Due to the faintness of the afterglow, a well-defined SED, corrected for host-galaxy light, cannot be constructed.

Energy budget: no redshift is known for GRB 081226A. Assuming a redshift of $z=0.5$ and using the data and the 


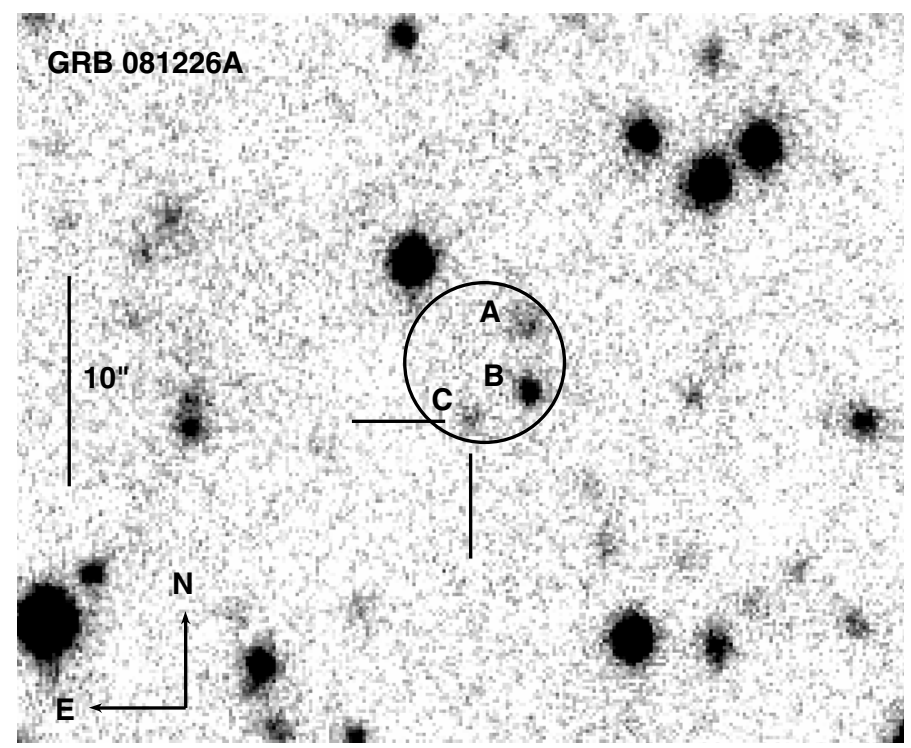

Fig. 1. White-band image of the field of GRB 081226A. Inside the $90 \%$ c.l. XRT error circle $(r=3$ "' 8$)$ lie three objects $(\mathrm{A}, \mathrm{B}, \mathrm{C})$. The position of the afterglow is indicated (C). In order to go deep, all GROND images of the first and the second epoch have been combined.

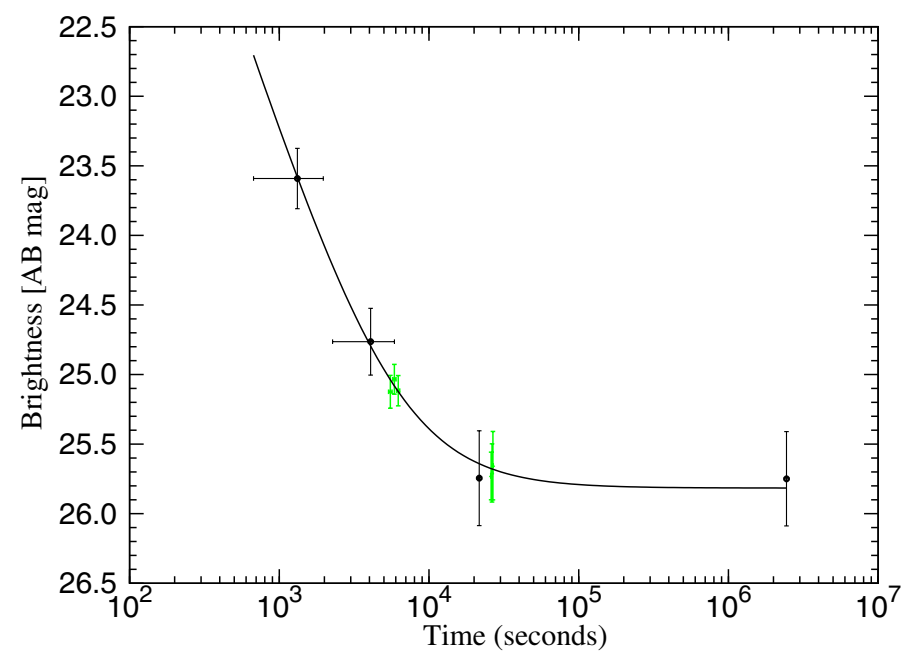

Fig. 2. GROND $r^{\prime}$-band light curve of the afterglow of GRB 081226A fitted with a single power-law plus host galaxy component. Overplotted in green are the Gemini-S/GMOS $r^{\prime}$-band data (Table A.1). No corrections for the slightly different filters have been performed.

numerical approach from Butler et al. $(2007)^{3}$, we obtain an isotropic equivalent energy for this burst of $E_{\text {iso }}=2.0_{-0.5}^{+1.7} \times$ $10^{50} \mathrm{erg}$. If there is a jet break in the optical light curve, then it must have occurred after about $10 \mathrm{ks}$. Assuming that the density profile of the circumburst environment is decribed by an interstellar medium (ISM), for the jet half-opening angle (e.g., Frail et al. 2001; Lu et al. 2012) we have

$$
\begin{aligned}
\Theta_{\text {jet }}= & 0.057 \mathrm{rad}\left(\frac{t_{\mathrm{b}}}{1 \text { day }}\right)^{3 / 8}\left(\frac{1+z}{2}\right)^{-3 / 8}\left(\frac{E_{\text {iso }}}{10^{53} \mathrm{erg}}\right)^{-1 / 8} \\
& \times\left(\frac{\eta_{\gamma}}{0.2}\right)^{1 / 8}\left(\frac{n}{0.1 \mathrm{~cm}^{-3}}\right)^{1 / 8} .
\end{aligned}
$$

\footnotetext{
3 http://astro.berkeley.edu/ nat/swift/bat_spec_table. html
}

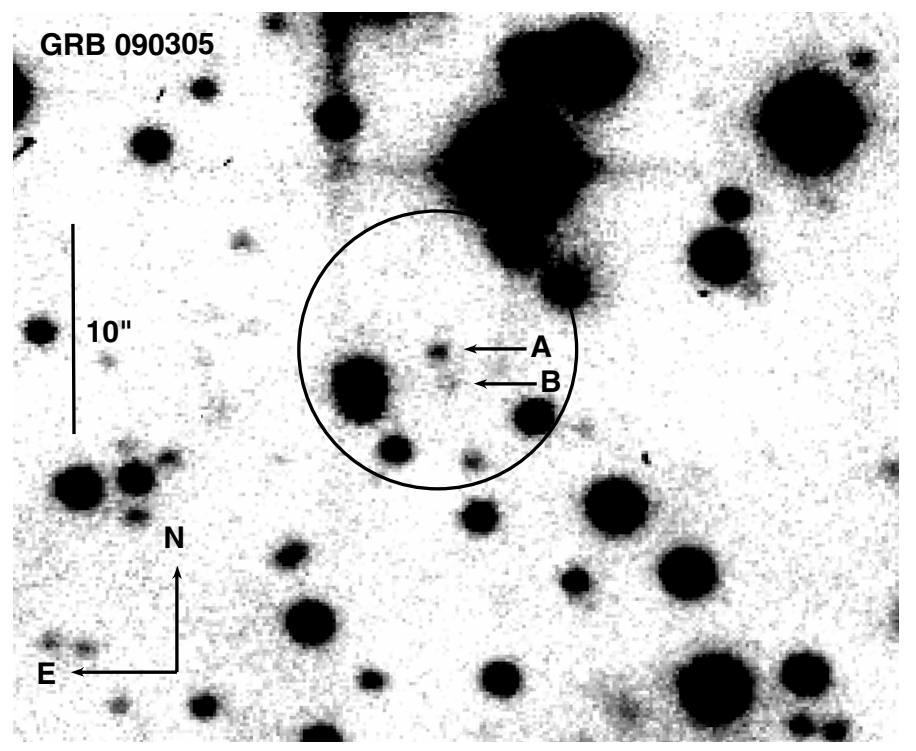

Fig. 3. Field of GRB 090305: the optical afterglow (A) and the object closest to it (B). Shown here is the $g^{\prime} r^{\prime} i^{\prime} z^{\prime}$-combined (white-band) image taken between $4 \mathrm{ks}$ to $7 \mathrm{ks}$ after the burst. The circle is just drawn to guide the eye; there is no independent Swift/XRT position (Beardmore et al. 2009c).

Adopting a radiative efficiency of 0.2 and scaling the results to a low gas density of $0.01 \mathrm{~cm}^{-3}$ as might be implied for a neutron star merger, we obtain $\Theta_{\text {jet }} \gtrsim 2.6_{-0.2}^{+0.1} \times(n / 0.01)^{1 / 8} \mathrm{deg}$ and a beaming-corrected energy of $E_{\text {cor }} \gtrsim 2.1_{-0.4}^{+1.3} 10^{47} \times$ $(n / 0.01)^{2 / 8} \mathrm{erg}$. There are no X-ray data for $t>10 \mathrm{ks}$ that could yield further evidence for a possible break in the afterglow light curve (Evans et al. 2010).

Host galaxy: the underlying host galaxy (C) is very faint and only visible in the $g^{\prime}, r^{\prime}$ second-epoch images $\left(g^{\prime}=25.88 \pm\right.$ $\left.0.24, r^{\prime}=25.79 \pm 0.34\right)$. The offset of the afterglow from its center is $\lesssim 0$ '. $^{\prime}$. For an assumed redshift of $z=0.5$, this corresponds to $\lesssim 3 \mathrm{kpc}$. No statement can be made about the morphological type of this galaxy.

\subsubsection{GRB 090305: Discovery of a jet break}

Afterglow light curve: GROND started observing the field 30 min after the Swift/BAT trigger and was on target for $1.5 \mathrm{~h}$. The fading optical afterglow (Cenko et al. 2009; Berger \& Kelson 2009) is detected in all optical bands but it is not seen in the NIR (Table 4).

Gemini-S/GMOS observed from $1.5 \mathrm{ks}$ to $7.5 \mathrm{ks}$ after the burst in $g^{\prime}, r^{\prime}, i^{\prime}$ and discovered the afterglow (Cenko et al. 2009). No detailed light curve data have been published so far, with $i^{\prime}$-band data affected by strong fringing. Figure 4 shows the result of the simultaneous fit of all data (GROND and Gemini) using a broken power-law with the Gemini data overplotted. The fit finds a break in the light curve at $t_{\mathrm{b}}=6.6 \pm 0.4 \mathrm{ks}$, a pre-break decay slope of $\alpha_{1}=0.56 \pm 0.04$, and a post-break decay slope of $\alpha_{2}=2.29 \pm 0.60$. The pre-break decay slope is rather shallow but not unusual (e.g., Zeh et al. 2006). There is no X-ray light curve available for this afterglow (Beardmore et al. 2009b).

$S E D$ : by fitting the Gemini $g^{\prime}$ - and $r^{\prime}$-band data, together with the GROND $g^{\prime} r^{\prime} i^{\prime} z^{\prime}$-band data, we find a spectral slope of $\beta_{\text {opt }}=0.52 \pm 0.15\left(\chi^{2} /\right.$ d.o.f. $\left.=0.66\right)$. No evidence for color evolution was found. Applying the $\alpha-\beta$ relations, there is no solution with $p>2$ for the pre-jet break phase; the light curve decay is 


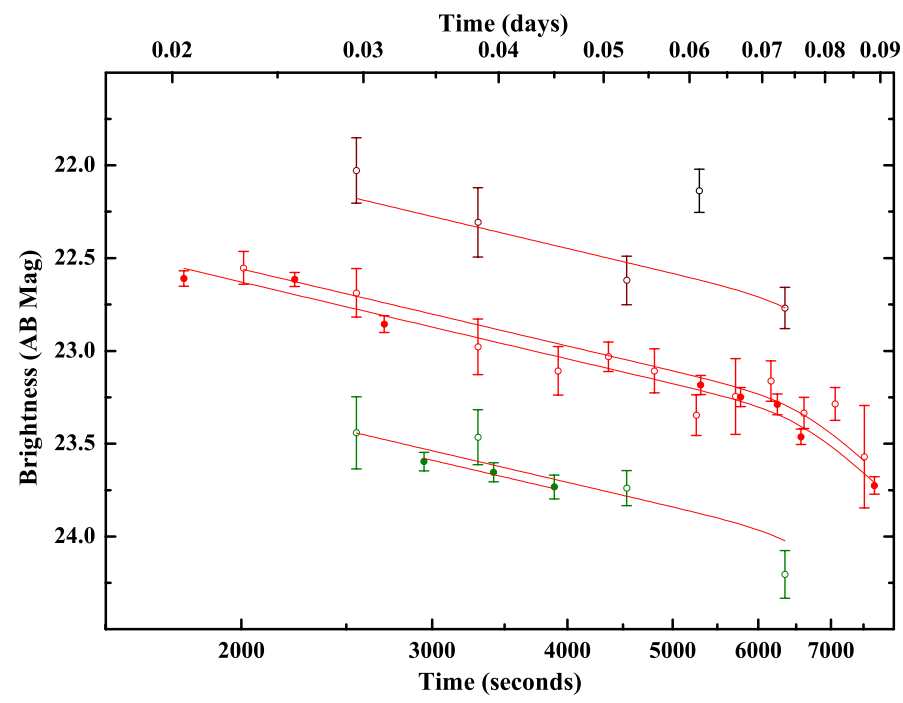

Fig. 4. Gemini and GROND light curve of the optical afterglow of GRB 090305. All data are fit simultaneously. Open circles are GROND, while filled circles are Gemini. Color coding: green $g^{\prime}$ band (shifted by $+0.5 \mathrm{mag}$ ), red $r^{\prime}$ band, brown $i^{\prime}$ band (shifted by -0.5 mag), black $z^{\prime}$ band (shifted by $-1 \mathrm{mag}$ ).

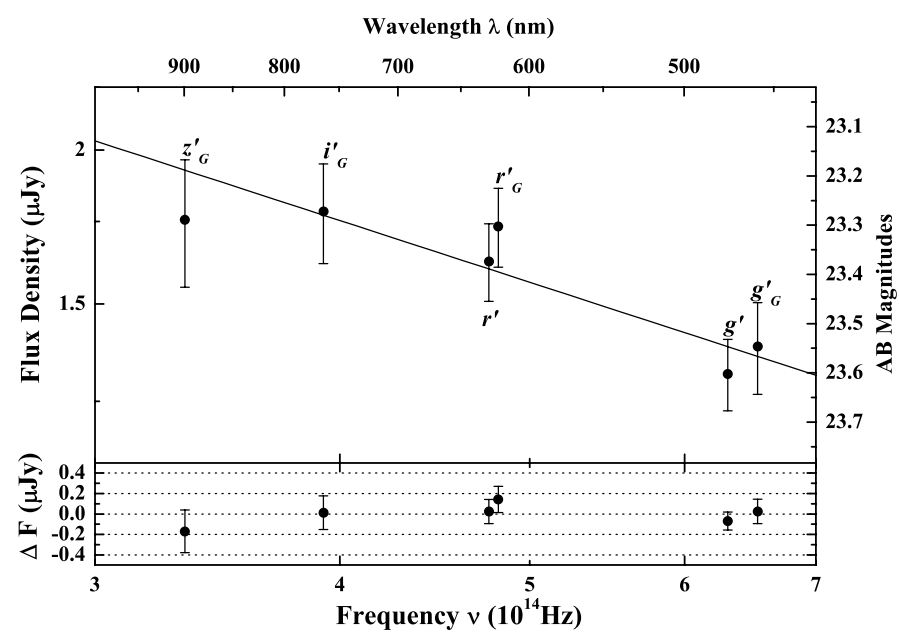

Fig. 5. GROND SED of the afterglow of GRB 090305 at $6 \mathrm{ks}$ after the burst, after correction for Galactic extinction. Index $G$ stands for GROND.

too shallow at that time (Table 2). On the other hand, the observed spectral slope suggests that between about $2 \mathrm{ks}$ and $8 \mathrm{ks}$ it was $v_{\mathrm{opt}}<v_{\mathrm{c}}$, because then $p=2 \beta+1=2.04 \pm 0.32$, a standard value. Possibly, the deduced shallow $\alpha_{1}$ indicates that at early times the evolution of the light curve was affected by re-brightening episodes or energy injections. No decision can be made between a wind and an ISM model.

Energy budget: assuming a redshift of $z=0.5$ and following the same procedure as in Sect. 3.1.1, we find $E_{\text {iso }}=2.1_{-0.7}^{+1.7} \times$ $10^{50} \mathrm{erg}$. The observed break time, if interpreted as a jet break in an ISM medium (Eq. (1)), leads to a jet half-opening angle of $\Theta_{\text {jet }}=2.2_{-0.1}^{+0.2} \times(n / 0.01)^{1 / 8} \mathrm{deg}$ and a beaming-corrected energy release of $E_{\text {cor }}=1.6_{-0.4}^{+0.9} \times 10^{47} \times(n / 0.01)^{2 / 8} \mathrm{erg}$.

Host galaxy: at the position of the optical transient, there is no evidence for an underlying host galaxy in any band; only upper limits can be given $\left(g^{\prime} r^{\prime} i^{\prime} z^{\prime} J H K_{\mathrm{s}}>25.7,26.0,24.5,24.2\right.$, $22.4,22.0,20.6)$. The object closest to the optical afterglow is a faint source at a distance of 1".4 (object B; see Fig. 3). This
Table 2. GRB 090305: predicted $\beta$ based on the $\alpha-\beta$ relations using $\alpha_{1}=0.56 \pm 0.04$ and $\alpha_{2}=2.29 \pm 0.60$.

\begin{tabular}{lccc}
\hline \hline Afterglow model & $\beta(\alpha)$ & Predicted $\beta$ & $p$ \\
\hline ISM, iso, case 1 & $\left(2 \alpha_{1}+1\right) / 3$ & $0.71 \pm 0.06$ & $1.42 \pm 0.12$ \\
ISM, iso, case 2 & $2 \alpha_{1} / 3$ & $0.38 \pm 0.06$ & $1.76 \pm 0.12$ \\
ISM, jet, case 1 & $\alpha_{2} / 2$ & $1.15 \pm 0.32$ & $2.30 \pm 0.50$ \\
ISM, jet, case 2 & $\left(\alpha_{2}-1\right) / 2$ & $0.65 \pm 0.32$ & $2.30 \pm 0.50$ \\
\hline wind, iso, case 1 & $\left(2 \alpha_{1}+1\right) / 3$ & $0.71 \pm 0.06$ & $1.42 \pm 0.12$ \\
wind, iso, case 2 & $\left(2 \alpha_{1}-1\right) / 3$ & $0.05 \pm 0.06$ & $1.10 \pm 0.12$ \\
wind, jet, case 1 & $\alpha_{2} / 2$ & $1.15 \pm 0.32$ & $2.30 \pm 0.50$ \\
wind, jet, case 2 & $\left(\alpha_{2}-1\right) / 2$ & $0.65 \pm 0.32$ & $2.30 \pm 0.50$ \\
\hline
\end{tabular}

Notes. Case 1 stands for $v>v_{\mathrm{c}}$, case 2 for $v<v_{\mathrm{c}}$. In the former case, the power-law index of the electron distribution function is given by $p=2 \beta$, whereas in the latter case $p=2 \beta+1$ (Sari et al. 1999).

object is only detected in the GROND $i^{\prime}$ band with a magnitude of $24.1 \pm 0.2$. Object B is also detected in Gemini $r^{\prime}$ band data taken ten days after the burst at a magnitude of $26.0 \pm 0.1$. It was also imaged with VLT/FORS in $R_{\mathrm{c}}$ (program ID 082.D-0451; PI: A. Levan).

Following the procedure described in Bloom et al. (2002) and Perley et al. (2009), the probability $p$ to find a galaxy as bright as object B within 1'." 4 distance from the afterglow is about $7 \%$. Formally, this small probability makes B a host galaxy candidate. If its observed color $\left(r^{\prime}-i^{\prime}=2.3 \pm 0.2 \mathrm{mag}\right)$ is due to the redshifted stellar $4000 \AA$ bump, its redshift is around $z=0.5^{4}$. For $z=0.5$, the projected distance of the afterglow from object B would be $8.5 \mathrm{kpc}$. There are, however, at least two more galaxies within $r=7$ arcsec around the position of the afterglow for which the $p$ value is of similar or smaller amount (see also Berger 2010a).

\subsubsection{GRB 090927: a wind medium?}

Observations: GROND started observations about $17 \mathrm{~h}$ after the burst and continued for $1.5 \mathrm{~h}$. A second-epoch observation was performed the following night for about $1 \mathrm{~h}$. Both observing runs were affected by bad seeing (2'.3). The afterglow was clearly fading in all GROND optical bands, while it was not detected in the NIR.

Afterglow light curve: the GROND $r^{\prime}$-band light curve can be fitted with a single power-law that has a slope of $\alpha=1.32 \pm$ $0.14\left(\chi^{2} /\right.$ d.o.f. $=0.39$; Fig. 6$)$, which is also in agreement with the results from the Faulkes Telescope South (Cano et al. 2009) and the VLT (Levan et al. 2009a). However, the first two $R$-band data points from the Zadko telescope (Klotz et al. 2009; see appendix) lie about 1 mag below the extrapolated fit (but also have large errors). Those data suggest that the optical flux was nearly constant between two and four hours after the burst. At the same time, the X-ray light curve shows strong fluctuations but seems to be in a plateau phase.

Assuming a single power-law decay for the X-ray light curve, we obtain $\alpha_{\mathrm{X}}=1.30 \pm 0.07$ for $t>20 \mathrm{ks}$. On the other hand, the outlier at $70 \mathrm{ks}$ could also be interpreted as evidence for a break in the X-ray light curve. However, the light curve decay after the break is then too shallow for a post-jet break decay slope. We thus conclude that also the X-ray afterglow is best described by pre-jet break evolution up to the end of the

\footnotetext{
4 Assuming that this is the GRB host galaxy, this color cannot be the Lyman break because the afterglow was detected in the $g^{\prime}$ band (Cenko et al. 2009).
} 


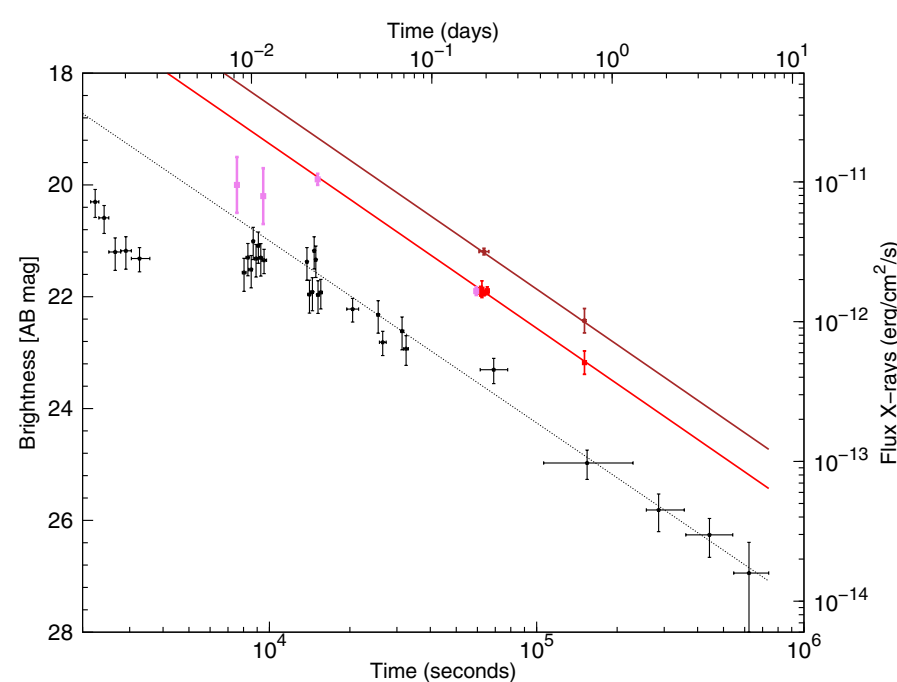

Fig. 6. The $r^{\prime}, i^{\prime}$-band light curve of the optical afterglow of GRB 090927 (the $i^{\prime}$-band is shifted by -0.6 mag; Table A.4). Overplotted are also $R$-band data reported in GCN Circulars (Klotz et al. 2009; Cano et al. 2009; Levan et al. 2009a; in violet) as well as the X-ray data (Evans et al. 2010).

XRT observations. A decay slope of 1.3 is in agreement with the ensemble statistics of pre-jet break decay slopes for long-burst afterglows (Zeh et al. 2006).

$S E D$ : the SED of the afterglow was constructed by combining all GROND data taken from 64 ks to $66 \mathrm{ks}$ after the trigger, when the seeing was best (about $2^{\prime \prime}$ ). It is best fit by a powerlaw with no extinction in the host galaxy $\left(A_{V}^{\text {host }}=0\right.$; Fig. 7). The spectral slope is $\beta_{\text {opt }}=0.41 \pm 0.16$. The $\alpha-\beta$-relations then imply that at the time of the GROND observations it was $v_{\mathrm{opt}}<v_{\mathrm{c}}$ (Table 3). The spectral slope $\beta_{\mathrm{X}}$ in the $\mathrm{X}$-ray band during this time period was $1.2 \pm 0.2$ (Evans et al. 2010), which in combination with the spectral slope in the optical points to $v_{\mathrm{opt}}<v_{\mathrm{c}}<v_{\mathrm{X}}$ and prefers a wind over an ISM model. For the pre-jet break phase this order in frequencies implies $\alpha_{X}-\alpha_{\text {opt }}= \pm 0.25$ ( - for a wind, + for an ISM), while we measure a difference of $-0.02 \pm 0.17$, not favoring any of both models.

Figure 8 shows the optical-to-X-ray SED of the afterglow at $t=65 \mathrm{ks}$. Using a Galactic $N_{\mathrm{H}}=2.9 \times 10^{20} \mathrm{~cm}^{-2}$, for the given redshift ( $z=1.37$; Levan et al. 2009a) the fit finds no evidence for host extinction (SMC dust; $A_{V}^{\text {host }}=0.02 \pm 0.02 \mathrm{mag}$ ), a spectral slope $\beta_{\mathrm{opt}}=0.57_{-0.10}^{+0.17}$, and a break energy of $42 \mathrm{eV}$ $\left(\chi^{2} /\right.$ d.o.f. $\left.=196 / 229=0.85\right)$. A fit with a single power-law is worse, confirming that $v_{\mathrm{opt}}<v_{\mathrm{c}}<v_{\mathrm{X}}$. 2003)

Energy budget: assuming a wind model, it is (Bloom et al.

$$
\begin{aligned}
\Theta_{\text {jet }}= & 0.169 \mathrm{rad}\left(\frac{t_{\mathrm{b}}}{1 \mathrm{day}}\right)^{1 / 4}\left(\frac{1+z}{2}\right)^{-1 / 4}\left(\frac{E_{\mathrm{iso}}}{10^{52} \mathrm{erg}}\right)^{-1 / 4} \\
& \times A_{\star}^{1 / 4}\left(\frac{\eta_{\gamma}}{0.2}\right)^{1 / 4},
\end{aligned}
$$

where $A_{\star}$ is the wind density parameter (Chevalier \& Li 2000) and, similar to Eq. (1), we have introduced a radiative efficiency $\eta_{\gamma}$. For a jet break time of $t_{\mathrm{b}}>6 \times 10^{5} \mathrm{~s}$ (as implied by the X-ray data), then for $z=1.37$ and $\eta_{\gamma}=0.2$, with $E_{\text {iso }}=4.5_{-2.0}^{+3.0} \times$ $10^{51} \mathrm{erg}$, we find $\Theta_{\text {jet }} \gtrsim 12 \pm 2 \mathrm{deg}$ and $E_{\text {cor }} \gtrsim 1.0_{-0.2}^{+0.3} \times 10^{50} \mathrm{erg}$. An ISM model (Eq. (1)) gives $\Theta_{\text {jet }}=7.0_{-0.4}^{+0.5} \times(n / 0.01)^{1 / 8} \mathrm{deg}$ and $E_{\text {cor }}=3.4_{-1.2}^{+1.5} \times 10^{49} \times(n / 0.01)^{2 / 8} \mathrm{erg}$.

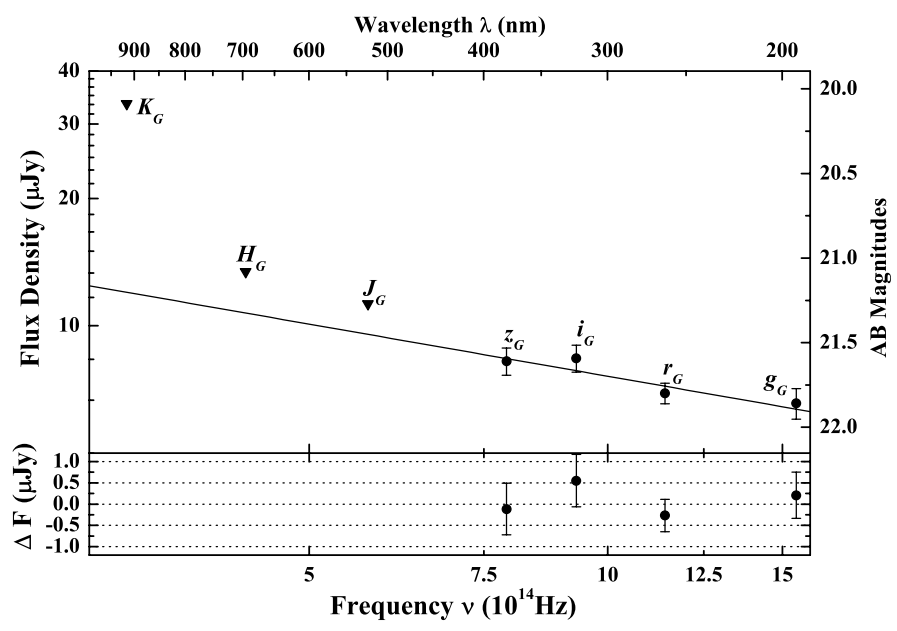

Fig. 7. SED of the afterglow of GRB 090927 at $t=65 \mathrm{ks}$ (from $g^{\prime}$ to $K_{\mathrm{s}}$ ). It is best fitted by a power-law with no evidence for extinction in the host galaxy. Note that the NIR bands are only upper limits.

Table 3. GRB 090927: predicted $\beta$ based on the $\alpha-\beta$ relations using $\alpha=1.32 \pm 0.14$ (for details, see Table 2).

\begin{tabular}{lcccc}
\hline \hline Afterglow model & $\beta(\alpha)$ & $\beta_{\mathrm{opt}}$ & $p$ & $\beta_{\mathrm{X}}$ \\
\hline ISM, iso, case 1 & $\left(2 \alpha_{1}+1\right) / 3$ & $1.21 \pm 0.09$ & $2.42 \pm 0.18$ & $1.20 \pm 0.05$ \\
ISM, iso, case 2 & $2 \alpha_{1} / 3$ & $0.88 \pm 0.09$ & $2.76 \pm 0.18$ & $0.87 \pm 0.05$ \\
\hline wind, iso, case 1 & $\left(2 \alpha_{1}+1\right) / 3$ & $1.21 \pm 0.09$ & $2.42 \pm 0.18$ & $1.20 \pm 0.05$ \\
wind, iso, case 2 & $\left(2 \alpha_{1}-1\right) / 3$ & $0.55 \pm 0.09$ & $2.10 \pm 0.18$ & $0.53 \pm 0.05$ \\
\hline
\end{tabular}

Notes. $p$ is given based on $\beta_{\text {opt }}$.

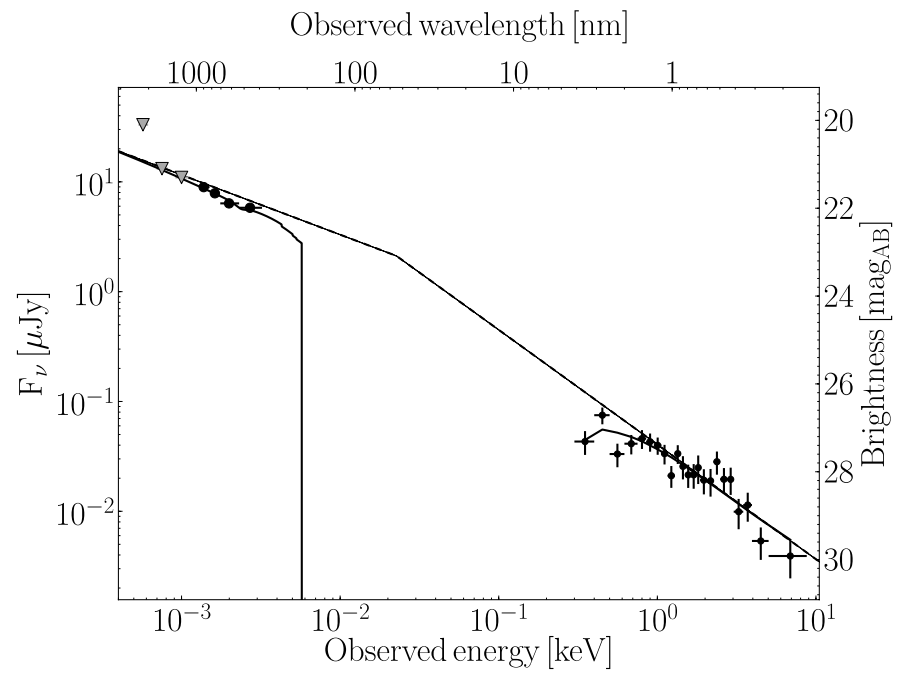

Fig. 8. Optical-to-X-ray SED of the afterglow of GRB 090927 at $t=$ $65 \mathrm{ks}$.

Host galaxy: observations performed two years after the trigger show no evidence of a host galaxy at the position of the optical transient down to deep upper limits $\left(g^{\prime} r^{\prime} i^{\prime} z^{\prime} J H K_{\mathrm{s}}>25.2\right.$, $25.2,24.5,24.2,22.3,21.6,20.4)$. The late-epoch data reveal that there are two objects, $\mathrm{A}$ and $\mathrm{B}$, within a radius of $10 \operatorname{arcsec}$ centered at the position of the optical afterglow (Fig. 9). Both objects are clearly extended. If one of them is the host, the projected offset of the burst was 6.'5 and 7".5, respectively. For a redshift of $z=1.37$ (Levan et al. 2009a), this corresponds to a projected distance of $55 \mathrm{kpc}$ and $63 \mathrm{kpc}$, respectively. If the 


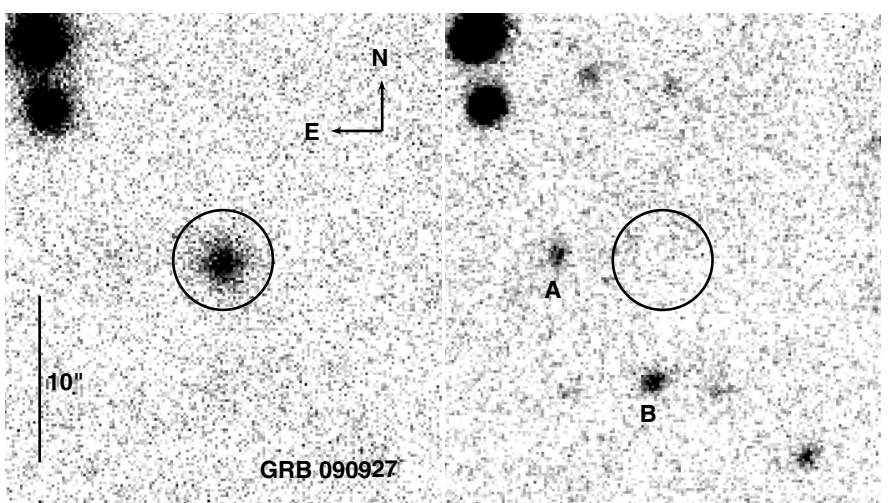

Fig. 9. Finding chart of the field of GRB 090927 (GROND $g^{\prime} r^{\prime} i^{\prime} z^{\prime}$-band combined). Left: first-epoch detection of the afterglow with GROND. Right: deep, late-epoch observation of the field in June 2011. The circle (2.'5 in radius), drawn to guide the eye, is centered at the position of the optical afterglow. A and B label the two galaxies nearest to the afterglow.

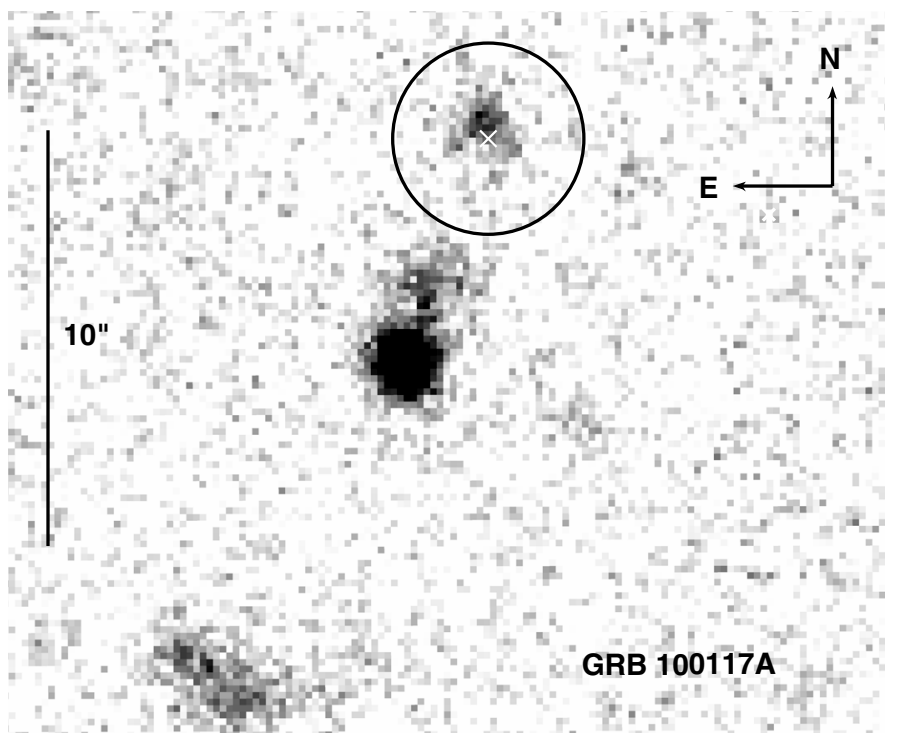

Fig. 10. Combined GROND $g^{\prime} r^{\prime} i^{\prime} z^{\prime}$-band (white) image of the field of GRB 100117A taken half a year after the burst. The circle is just drawn to guide the eye. It is centered at the position of the optical transient discovered by Fong et al. (2011) and circumscribes the GRB host galaxy.

progenitor of GRB 090927 was a collapsar, this large distance rules out that $\mathrm{A}$ or $\mathrm{B}$ is the putative host.

\subsubsection{GRB 100117A: determination of the afterglow decay slope}

Observations: GROND started observing the field of GRB 100117A $3.5 \mathrm{~h}$ after the GRB trigger and was on target for one hour (Fig. 10). The host galaxy flux was measured half a year later.

Afterglow: the optical afterglow on top of its host galaxy was discovered by Fong et al. (2011). During the first night, we measure for the host plus afterglow a $g^{\prime}, r^{\prime}$-band magnitude of $24.37 \pm 0.25,23.72 \pm 0.18$. However, in the late-epoch data $g^{\prime}, r^{\prime}=25.44 \pm 0.37,24.60 \pm 0.35$, resulting in a decay between both epochs of $1.07 \pm 0.45 \mathrm{mag}$ and $0.88 \pm 0.39 \mathrm{mag}$, respectively.

The second-epoch data can be used to remove the host galaxy flux from the first-epoch data. Based on this result, we

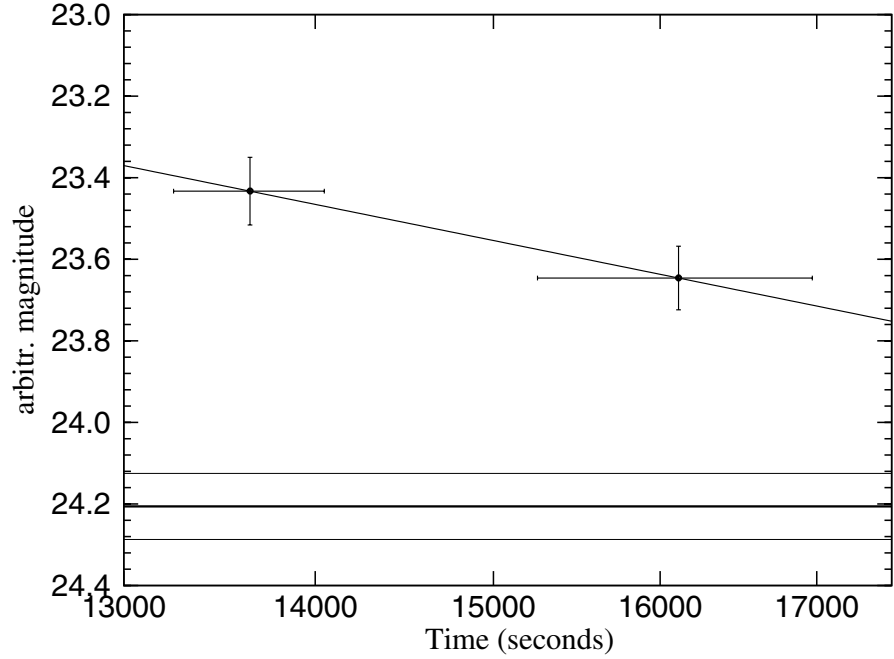

Fig. 11. Combined GROND $g^{\prime} r^{\prime} i^{\prime}$ white-band light curve of the decaying afterglow of GRB 100117A, centered on galaxy (Fig. 10). The host galaxy magnitude is shown as a straight line, including the $1 \sigma$ error (measured by GROND eight months after the burst). Note that the $y$-axis shows arbitrarily magnitudes.

obtain an afterglow magnitude of $r^{\prime} \sim 24.3$ during our firstepoch observations at a mean time of $t=4.3 \mathrm{~h}$. We can estimate the decay slope of the afterglow light curve by comparing this result with the $r$-band detection of the afterglow by Fong et al. (2011) $8.3 \mathrm{~h}$ after the burst. This gives $\alpha \sim 1.3$, assuming no color transformation between both filters. This result is confirmed by combining the GROND $g^{\prime} r^{\prime} i^{\prime}$ images into a white band. Figure 11 shows the corresponding light curve of the afterglow during the first night, providing a slope of $\alpha=1.2$. This indicates that during this time period the afterglow was still in its pre-jet break phase.

Energy budget: Swift/XRT data do not cover the time period when GROND and Fong et al. (2011) were observing. The last X-ray detection is at $477_{-57}^{+101} \mathrm{~s}$ after the trigger (Evans et al. 2010). Particularly because the very last XRT data point at around $0.5 \mathrm{~d}$ is only an upper limit, optical and XRT data cannot be compared. If the afterglow was in the pre-jet break decay phase until at least $8.3 \mathrm{~h}$ after the burst, in combination with the observed isotropic equivalent energy of $E_{\text {iso }}=$ $51.0_{-0.1}^{+0.1} \times 10^{50} \mathrm{erg}$ (Kann et al. 2011) and a redshift of $z=0.92$ (Fong et al. 2011), the lower limit on the jet half-opening angle is (Eq. (1)) $\Theta_{\text {jet }}=2.4 \times(n / 0.01)^{1 / 8} \mathrm{deg}$ and $E_{\text {cor }} \gtrsim 4.6 \times 10^{48} \times$ $(n / 0.01)^{2 / 8} \mathrm{erg}$.

Host galaxy: our data do not allow us to measure the offset of the afterglow from its host galaxy center; Fong et al. (2011), using their Gemini-N/GMOS observations, obtained $60 \pm 40$ mas, corresponding to $0.5 \pm 0.3 \mathrm{kpc}$.

\subsection{GRBs with no afterglow detected by GROND}

The results for the 14 out of 20 GRBs where GROND could not detect the afterglow are summarized in Table 4. In most cases, we were on target within some hours after the burst. In all cases, deep upper limits can be provided, in particular in the NIR, where we reach down to $J=22.7, H=22.0$, and $K_{\mathrm{s}}=21.2$. The individual observations by GROND are described in detail in the appendix. However, of particular interest are two events (GRB 080919, 100702A), where observations started less than $10 \mathrm{~min}$ after the trigger. 
Table 4. Summary of the $3 \sigma$ upper limits for the short-burst afterglows not detected with GROND based on first-epoch data (AB magnitudes).

\begin{tabular}{|c|c|c|c|c|c|c|c|c|c|c|c|c|c|}
\hline (1) & $\begin{array}{l}\text { GRB } \\
(2) \\
\end{array}$ & $\begin{array}{l}t_{\mathrm{GRB}} \\
(\mathrm{UT}) \\
(3) \\
\end{array}$ & (4) & $\begin{array}{c}t_{\text {start }}^{\text {obs }} \\
(\mathrm{UT}) \\
(5) \\
\end{array}$ & $\begin{array}{l}\text { Mean } \\
\text { (UT) } \\
(6) \\
\end{array}$ & $\begin{array}{c}<d t> \\
\text { (hh:mm:ss) } \\
\text { (7) }\end{array}$ & $\begin{array}{l}g^{\prime} \\
(8)\end{array}$ & $\begin{array}{l}r^{\prime} \\
(9)\end{array}$ & $\begin{array}{c}i^{\prime} \\
(10)\end{array}$ & $\begin{array}{l}z^{\prime} \\
(11)\end{array}$ & $\begin{array}{c}J \\
(12)\end{array}$ & $\begin{array}{c}H \\
(13)\end{array}$ & $\begin{array}{l}K_{\mathrm{s}} \\
(14)\end{array}$ \\
\hline 1 & 070729 & $00: 25: 53$ & 1 & 29-Jul.-2007, 07:09:53 & $09: 13: 33$ & $08: 47: 40$ & 24.5 & 24.7 & 24.4 & 24.3 & 22.7 & 21.8 & - \\
\hline 2 & 071112B & $18: 23: 31$ & 2 & 13-Nov.-2007, 00:11:25 & $00: 49: 15$ & $06: 25: 44$ & 24.6 & 24.4 & 23.8 & 23.5 & 21.6 & 20.7 & 20.0 \\
\hline 3 & 071227 & $20: 13: 47$ & 3 & 28-Dec.-2007, 00:20:05 & $00: 23: 14$ & 04:09:27 & - & 20.6 & 20.0 & 20.4 & 20.0 & 19.8 & 19.4 \\
\hline 4 & 080905A & $11: 58: 54$ & 4 & 06-Sep.-2008, 05:22:14 & $05: 27: 53$ & $17: 28: 59$ & 23.0 & 22.8 & 22.3 & 21.9 & 20.4 & 19.9 & 19.6 \\
\hline \multirow{4}{*}{5} & 080919 & $00: 05: 13$ & 5 & 19-Sep.-2008, 00:13:31 & $00: 16: 52$ & $00: 11: 39$ & - & - & - & - & 19.6 & 19.4 & 19.3 \\
\hline & & & & 00:13:31 & $00: 20: 34$ & $00: 15: 21$ & - & - & - & - & 19.8 & 19.5 & 19.5 \\
\hline & & & & 00:28:09 & $00: 39: 16$ & 00:34:03 & - & - & - & - & 19.8 & 19.7 & - \\
\hline & & & & $00: 53: 11$ & $01: 22: 37$ & $01: 17: 24$ & 23.5 & 22.7 & 22.2 & 21.9 & 19.7 & 19.7 & 19.8 \\
\hline 7 & 081226B & 12:13:11 & 6 & 27-Dec.-2008, 01:30:14 & $02: 00: 55$ & $13: 47: 44$ & 25.5 & 25.2 & 24.3 & 23.9 & 22.0 & 21.5 & 20.5 \\
\hline 12 & 091109B & 21:49:03 & 7 & 10-Nov.-2009, 03:31:40 & $03: 45: 58$ & $05: 56: 55$ & 23.6 & 23.3 & 22.2 & 21.9 & 20.3 & 19.7 & 19.0 \\
\hline 13 & 091117A & $17: 44: 29$ & 8 & 19-Nov.-2009, 00:46:48 & $01: 13: 45$ & $31: 29: 16$ & 25.0 & 24.8 & 24.0 & 23.5 & 21.7 & 21.2 & 20.4 \\
\hline 15 & 100206A & 13:30:05 & 9 & 07-Feb.-2010, 00:33:50 & 01:09:43 & $11: 39: 28$ & 24.7 & 24.4 & 23.9 & 23.1 & 21.7 & 21.3 & 20.4 \\
\hline 16 & $100625 \mathrm{~A}$ & $18: 32: 28$ & 10 & 26-Jun.-2010, 06:13:15 & $06: 43: 04$ & $12: 10: 36$ & 23.6 & 23.1 & 22.8 & 22.9 & 21.8 & 21.2 & 20.3 \\
\hline 17 & $100628 \mathrm{~A}$ & $08: 16: 40$ & 11 & 29-Jun.-2010, 01:24:19 & 02:08:49 & $17: 52: 09$ & 24.2 & 24.5 & 23.9 & 23.9 & 22.6 & 22.0 & 21.2 \\
\hline \multirow[t]{3}{*}{18} & $100702 \mathrm{~A}$ & 01:03:47 & 12 & 02-Jul.-2010, 01:06:38 & 01:09:51 & 00:06:04 & 24.1 & 23.6 & 23.0 & 22.5 & 20.4 & 20.0 & 19.3 \\
\hline & & & & 01:06:38 & $01: 13: 27$ & 00:09:40 & 24.4 & 23.8 & 23.2 & 22.7 & 20.6 & 20.1 & 19.5 \\
\hline & & & & 01:21:04 & $01: 48: 27$ & $01: 44: 40$ & 24.9 & 24.1 & 23.5 & 23.0 & 20.6 & 20.3 & 19.8 \\
\hline 19 & 101129A & $15: 39: 32$ & 13 & 30-Nov.-2010, 06:20:25 & 07:11:30 & $15: 31: 58$ & 24.7 & 24.7 & 24.2 & 23.9 & 22.0 & 21.4 & 20.5 \\
\hline 20 & 101219A & $02: 31: 30$ & 14 & 19-Dec.-2010, 03:55:06 & $05: 09: 48$ & $02: 38: 18$ & 23.8 & 23.9 & 23.4 & 23.2 & 22.4 & 22.0 & 20.9 \\
\hline
\end{tabular}

Notes. Column 3: GRB trigger time (UT); Col. 5: time after the burst when the first optical $\mathrm{OB}^{5}$ was started; Col. 6: mean observing time; Col. 7: difference between Cols. 6 and 3 (always in hh:mm:ss). Columns 8-14: $3 \sigma$ upper limits. Notes to individual bursts: GRB 071227: just $1 \mathrm{OB}$ was taken in the first night (4 min), the $g^{\prime}$ band is not useful; GRB 080905A: just 1 OB was taken $(8 \mathrm{~min}), g^{\prime} r^{\prime} i^{\prime} z^{\prime}$ are calibrated based on GROND zeropoints; GRB 081226B: the optical upper limits refer to the southern 50\% of the error circle, the other part was not imaged in $g^{\prime} r^{\prime} i^{\prime} z^{\prime}$; GRB 100206A: this supercedes the information given in Nicuesa Guelbenzu et al. (2010). General: note that in the case of large BAT/IBIS error circles, the true limiting magnitudes of the afterglow might be 1 mag less deep than the limiting magnitudes of the images as given here.

References. 1 = Guidorzi et al. (2007c); 2 = Perri et al. (2007); 3 = Sakamoto et al. (2007a); 4 = Pagani et al. (2008b); $5=$ Preger et al. (2008b); $6=$ Mereghetti et al. (2008); $7=$ Grupe et al. (2009b); 8 = Cummings et al. (2009); $9=$ Krimm et al. (2010c); $10=$ Holland et al. (2010b); 11 = Immler et al. (2010); 12 = Siegel et al. (2010b); 13 = Cummings et al. (2010); 14 = Gelbord et al. (2010).

\subsubsection{GRB 080919}

GROND started observing the field 8 min after the burst. Due to a delay in secure XRT coordinates (Preger et al. 2008a), only the NIR images cover the afterglow position during the first $30 \mathrm{~min}$. Deep second-epoch observations were performed with GROND three years after the burst. Image subtraction was carried out between second- and first-epoch data in all bands, but no afterglow was found. Probably the main reason for this non-detection is the presence of a bright star inside the error circle, which makes it difficult to detect any faint transient despite the small XRT error circle (90\% c.l. radius $r=1{ }^{\prime \prime} 6$; Evans 2011b). Therefore, the upper limits we provide in Table 4 refer to isolated objects in the field, while the more reliable upper limits for the afterglow can be substantially less deep than reported there.

\subsubsection{GRB $100702 \mathrm{~A}$}

GROND started to observe the field $2.5 \mathrm{~min}$ after the burst. Inside the $90 \%$ c.l. XRT error circle ( $r=2$ ". 4 ; Siegel et al. 2010a), the GROND data reveal two bright objects (A, B) and two others (C, D) close by (Fig. 13; see also Malesani et al. 2010). Objects A and B have a point-like PSF and might be stars, while $\mathrm{C}$ and $\mathrm{D}$ might be galaxies.

Image subtraction and PSF photometry in each band was performed for all objects, but no evidence of variability was found in either the optical or the NIR bands; only upper limits can be provided for any afterglow (Table 4). Similar to GRB 080919, the upper limits refer to isolated objects in the field.

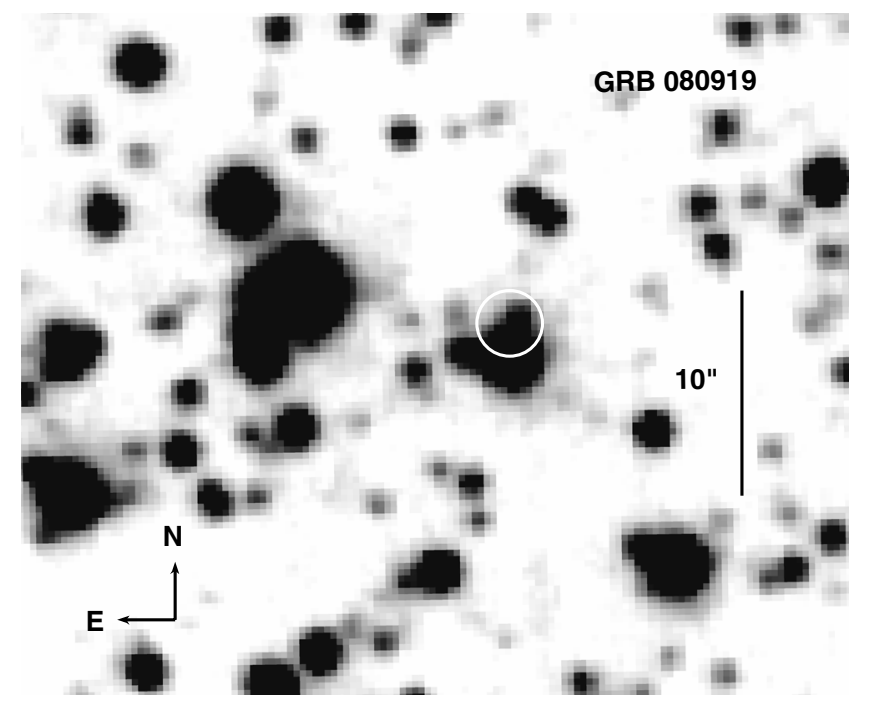

Fig. 12. Field of GRB 080919. The XRT error circle (radius $r=11^{\prime \prime} 6$ ) lies close to a relatively bright foreground star.

\section{Discussion}

Including our discovery of the afterglow and host galaxy of GRB 081226A, nine out of 20 short bursts in our sample have a discovered optical transient, while six have only a Swift/XRT and four have only a Swift/BAT or INTEGRAL/IBIS localization with no optical afterglow. Six of the nine bursts with 


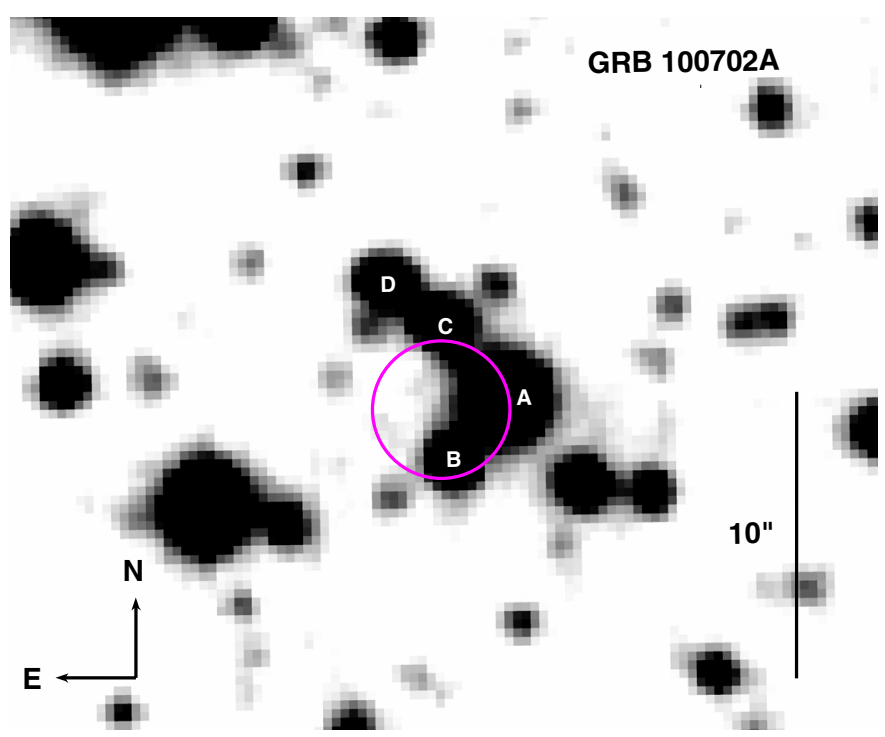

Fig. 13. Finding chart of the field of GRB 100702A in the GROND $J$ band. Shown also is the $90 \%$ c.l. XRT error circle $(r=2$ '. 4 ; Siegel et al. 2010a).

detected optical transient have a redshift reported in the literature. Additional redshift information comes from the identification of the host galaxies of GRBs 100206A (Cenko et al. 2010a; Perley et al. 2011), 100628A (Cenko et al. 2010b), and 101219A (Chornock \& Berger 2011). These redshifts range from $z=0.10$ (GRB 100628A) to $z=2.61$ (GRB 090426). Four of the nine bursts have a redshift smaller than 0.5 , which is a high percentage compared to the long-burst population; for more redshifts of short-bursts, see the compilations by Berger (2009), Kann et al. (2011).

The best-sampled light curves are those of GRB 090426 (Paper I) and GRB 090510 (Paper II), followed by (ordered by sampling quality) GRBs 090305, 081226A, 090927, and 100117A. Only the afterglow of GRB 090426 has NIR detections. In three cases, we find a clear break in the light curve, partly in combination with data obtained at other facilities. Two of these events (GRBs 090426, 090510) were imaged by GROND in the post-break decay phase only, and for GRB 090305 the data also included the pre-break phase. In principle, the three breaks might be interpreted as jet breaks, but for GRB 090510 the Swift/Ultraviolet Optical Telescope (UVOT) data suggest a different explanation, namely the passage of the injection frequency across the GROND bands (for details, see Kumar \& Barniol Duran 2010; de Pasquale et al. 2010d and Paper II). For the other three cases, the light curves can be fitted with a single power-law and, based on the deduced decay slope, observations were performed during the pre-jet break evolutionary phase. The light curve decay slopes as well as the spectral slopes are not different from what is known for the long-burst sample (Table 5).

\subsection{Optical luminosities}

In recent years, evidence has been mounting that the classical $T_{90}$ division between short and long GRBs is not transferable to a more physically inspired division between progenitor models. It seems that merging compact objects may result in high-energy emission on timescales far exceeding $T_{90}=2 \mathrm{~s}$, whereas conversely collapsar-triggered GRBs can be luminous short spikes with $T_{90, \text { rest }}<2$ s. This led Zhang et al. (2007) to propose,

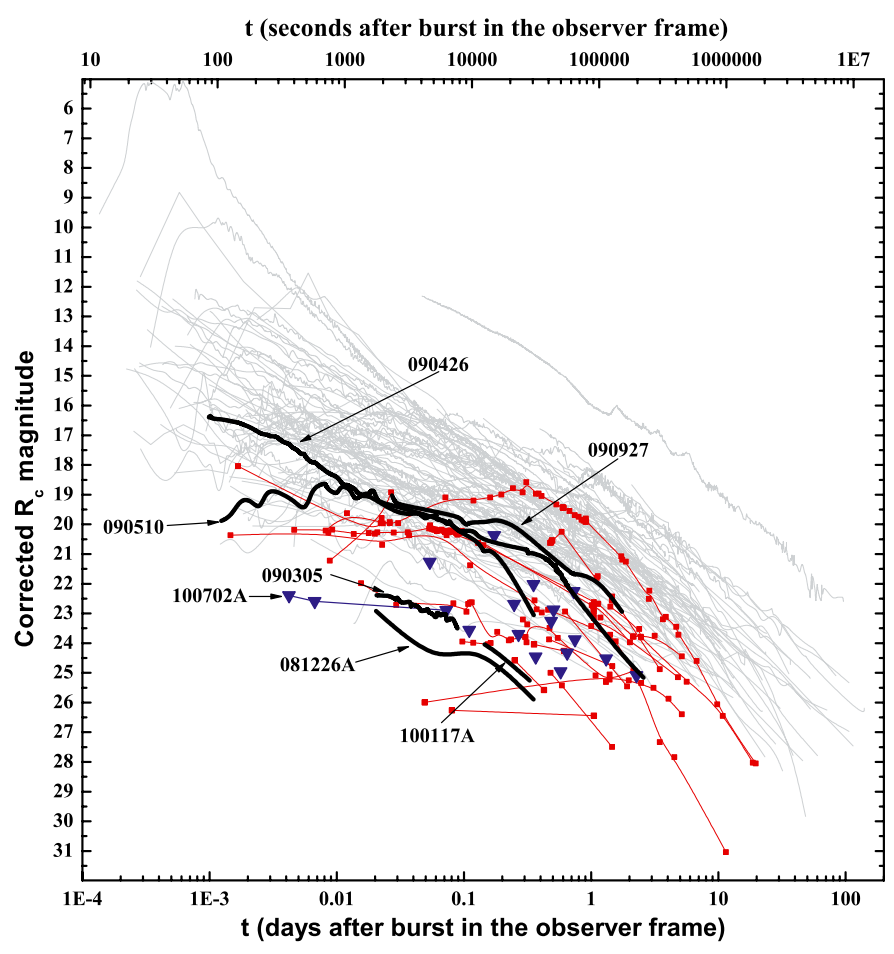

Fig. 14. Light curves of long and short GRB afterglows. These light curves have been corrected individually for Galactic foreground extinction following Schlegel et al. (1998), and, if possible, for host galaxy contribution. The thin gray lines are the long GRB sample of Kann et al. (2010). The red squares connected by splines represent the afterglow detections reported by Kann et al. (2011). The short GRB afterglows detected by GROND and presented in Papers I and II as well as this work are given as labeled thick black lines (they may include additional data beyond the GROND detections). Upper limits presented in this work (Table 4) are given as blue triangles. GRB 100702A is highlighted also because of its very early upper limits. The last data point for GRB 100117A is from Fong et al. (2011), the other data points as well as the data for GRBs 090305 and $081226 \mathrm{~A}$ are presented in this paper. Early data for GRB 090927 are taken from Klotz et al. (2009), Levan et al. (2009a), Cano et al. (2009), and Kuin \& Grupe (2009).

analogous to the designations of supernovae, that GRBs come in two types: Type I GRBs stem from the coalescence of massive compact objects, whereas Type II GRBs are associated with the core-collapse of massive stars. Zhang et al. (2009) studied the observational signatures of the two classes and devised a scheme to classify GRBs. Kann et al. (2011) studied a large sample of Type I candidate GRBs, adding the optical afterglow luminosity at late times as an additional criterion to discern the two classes, with Type I GRB afterglows being much less luminous than those of Type II GRBs.

So far, in this work, we have discussed the sample based on the classic $T_{90}$ division. What can the optical luminosity of the afterglows (or its upper limits) tell us about the likely progenitor systems? Figure 14 is a continuation of the plots shown in Kann et al. $(2006,2010,2011)$. Against the background of Type II GRB afterglow light curves (thin gray lines), we show Type I GRB afterglow detections as presented in Kann et al. (2011; red squares connected by splines, upper limits have been omitted for clarity) as well as the detected afterglows (thick black splines) and upper limits (downward-pointing blue triangles) derived by GROND both in this work and in Papers I and II.

In this plot, it is visible that the short GRB afterglows are less bright than the mean brightness of the long GRB afterglows, 
A\&A 548, A101 (2012)

Table 5. Summary of the data for the six bursts with optical afterglow detection by GROND.

\begin{tabular}{lcccccr}
\hline \hline GRB & $\alpha_{1}$ & $\alpha_{2}$ & $t_{\mathrm{b}}[\mathrm{ks}]$ & $\beta_{\text {opt }}$ & $\Theta_{\text {jet }}[\mathrm{deg}]$ & $E_{\text {cor }}[\mathrm{erg}]$ \\
\hline $081226 \mathrm{~A}$ & $1.3 \pm 0.2$ & - & $>10$ & - & $\gtrsim 2.6$ & $\gtrsim 2.1_{-0.4}^{+1.3} \times 10^{47}$ \\
090305 & $0.56 \pm 0.04$ & $2.29 \pm 0.60$ & $6.6 \pm 0.4$ & $0.52 \pm 0.15$ & $2.2 \pm 0.2$ & $1.6_{-0.4}^{+0.9} \times 10^{47}$ \\
090426 & $0.46 \pm 0.15$ & $2.43 \pm 0.19$ & $34.5 \pm 1.8$ & $0.76 \pm 0.14$ & $6.5 \pm 0.4$ & $4.2 \pm 1.4 \times 10^{48}$ \\
090510 & $1.13 \pm 0.11$ & $2.37 \pm 0.29$ & $1.6 \pm 0.4$ & $0.85 \pm 0.05$ & $\sim 1$ & $\sim 3 \times 10^{49}$ \\
090927 & $1.32 \pm 0.14$ & - & $>600$ & $0.57_{-0.10}^{+0.17}$ & $\gtrsim 12 \pm 2$ & $\gtrsim 1.0_{-0.2}^{+0.3} \times 10^{50}$ \\
$100117 \mathrm{~A}$ & $\sim 1.3$ & - & $>30$ & - & $\gtrsim 2.4$ & $\gtrsim 4.6 \times 10^{48}$ \\
\hline
\end{tabular}

Notes. GRB 090426: the light-curve parameters refer to the wide jet solution (see Paper I). GRB 090510: light-curve parameters of this burst are interpreted as a jet at very early times. $\alpha_{1}$ as well as $t_{\mathrm{b}}$ were taken from the optical fit as reported in de Pasquale et al. (2010d); $\Theta_{\text {jet }}$ and $E_{\text {cor }}$ were taken from He et al. (2011). For the other bursts, see this work. GRB 090927: constraints on the jet break time come from the X-ray data (Fig. 6). The results refer to a wind model.

with half of them (GRBs 090305, 10017A and 081226A) being as faint or fainter than the faintest so-far detected long GRB afterglows. A true comparison needs to account for the redshift and intrinsic extinction, though.

Figure 15 shows the light curves of the six short GRBs detected with GROND in the $z=1$ reference frame, corrected if possible for both distance and intrinsic reddening in the GRB host galaxy (Kann et al. 2006; Nardini et al. 2006). A redshift of $z=0.5$ and zero host extinction was assumed for all cases where these values are not known. Of the six afterglows, that of GRB 090426 is now seen to be the most luminous, followed by the ones of GRBs 090927 and 090510. Several arguments have already been put forward that the origin of 090426 was a collapsar event (see Paper I and references therein). Between about 0.01 and $0.1 \mathrm{~d}$ after the burst (measured in the GRB host frame), its magnitude (for the fixed distance a measure of the luminosity) was about 2 mag brighter than the magnitude of the optical afterglow of the other two events. The optical afterglow of GRB 090510, if due to a merger event, must be characterized as very luminous between $\sim 0.005$ and $0.1 \mathrm{~d}$ after the burst. Because of its emission in the $10-100 \mathrm{GeV}$ band and its outstandingly small jet half-opening angle of $\Theta_{\text {jet }} \lesssim 1^{\circ}$ (de Pasquale et al. 2010d; He et al. 2011; Kumar \& Barniol Duran 2010, Paper II; if correctly interpreted in this way), it was special in several other respects, too. The optical afterglow of GRB 090927 reached the luminosity of the afterglow of GRB 090426 at about $1 \mathrm{~d}$ after the burst, but its further evolution is unfortunately unknown. This moderately high optical luminosity along with significant lag and other spectral characteristics (Stamatikos et al. 2009) and a redshift beyond what is seen for Type I GRBs (Levan et al. 2009a) strongly indicate that GRB 090927 is also likely to be a Type II GRB. All other afterglows with GROND detections or GROND upper limits fall well within the Type I GRB sample.

Between the host time frame of about 0.01 and $0.1 \mathrm{~d}$, the three optical afterglows mentioned above (which have a measured redshift) were about $7 \pm 1$ mag brighter than the afterglows of GRBs 081226A, 090305, and 100117A (of which only the latter has a secure redshift $)^{6}$. For GRB 090510, the situation changes after about $0.1 \mathrm{~d}$, when the early break and following steep decay (Paper II) cause it to become much fainter than Type II GRB afterglows (see also Kann et al. 2011). From the perspective of optical luminosities, we therefore find additional evidence for a collapsar origin of GRB 090927, despite its short duration, whereas there is no evidence indicating that

\footnotetext{
6 If the redshift of the former two bursts is not 0.5 , as assumed here, but somewhere between 0.2 and 1.0, then the magnitude difference changes by about \pm 2 mag.
}

GRBs 090305 and 081226A are not members of the classical short/Type I GRB population. We note, though, that Panaitescu (2011) also discussed a collapsar origin for GRB 090510.

\subsection{Jet half-opening angles}

Observations of jet breaks in short-burst afterglow light curves are rather sparse, in the optical as well as in the X-ray band. In the optical band, the best-sampled cases are GRBs 090426 and 090510. However, the former burst is suspected to be owing to a collapsar explosion rather than to a merger event (e.g., Thöne et al. 2011), while the latter stands apart even from the long-burst sample because of its very small jet half-opening angle (He et al. 2011). The third member of this group is GRB 050709 with an estimated $\Theta_{\text {jet }} \sim 14 \mathrm{deg}$ (Fox et al. 2005), which is based on a very sparsely sampled light curve, however.

In the X-ray band, the observational situation is not much better. The best case might again be GRB 090510 (de Pasquale et al. 2010d), followed by GRBs 050724, 051221A, 061201, and 111020A. Unfortunately, the first burst (GRB 050724) allows only for an estimate of a lower limit on $\Theta_{\text {jet }}(\gtrsim 25 \mathrm{deg}$; Grupe et al. 2006; Malesani et al. 2007), while GRB 051221A relies on a well-sampled light curve (leading to $\Theta_{\text {jet }} \sim 4-8 \mathrm{deg}$; Burrows et al. 2006; Soderberg et al. 2006). The X-ray light curve of GRB 061201 is well-sampled, too (Stratta et al. 2007); again the observed break time is quite early ( $40 \mathrm{~min}$; $\Theta_{\text {jet }}=1-2 \mathrm{deg}$ ). Recently, Fong et al. (2012) reported on the X-ray light curve of the short burst $111020 \mathrm{~A}$, which showed a break at $2 \mathrm{~d}$, leading to an estimated $\Theta_{\text {jet }}=3-8 \mathrm{deg}$ for an assumed $z=0.5-1.5$ and $n \sim 0.01-0.1 \mathrm{~cm}^{-3}$.

Figure 16 shows the observed distribution of jet half-opening angles of long-bursts based on the compilation of Lu et al. (2012) compared to the short-burst sample (a similar plot has been recently shown by Fong et al. (2012). The latter contains the results summarized in Table 5, supplemented by GRBs 061006 $\left(\Theta_{\text {jet }} \sim 5 \mathrm{deg}\right), 070714 \mathrm{~B}\left(\Theta_{\text {jet }} \gtrsim 4 \mathrm{deg}\right)$, and $071227\left(\Theta_{\text {jet }} \gtrsim\right.$ $4 \mathrm{deg}$ ) taken from the compilation of Fan \& Wei (2011), but using $\eta_{\gamma}=0.2$ instead of 1.0 (i.e., multiplying their numbers by 0.8 ; Eq. (1)). At first view, this figure shows tentative evidence that short bursts have wider jet-opening angles than long bursts. Some caution is necessary, however. First of all, when calculating the jet half-opening angles, Lu et al. (2012) assumed $n=0.1 \mathrm{~cm}^{-3}$ and $\eta_{\gamma}=0.2$ throughout. Even though $\Theta_{\text {jet }}$ is only modestly sensitive to changes in both parameters (see Eq. (1)), gas densities derived for bursts based on multi-wavelength data show a spread from burst to burst by several orders of magnitude (e.g., Panaitescu \& Kumar 2001). Second, error bars in $\Theta_{\text {jet }}$ are not taken into account in the histogram. Similarly, our standard 


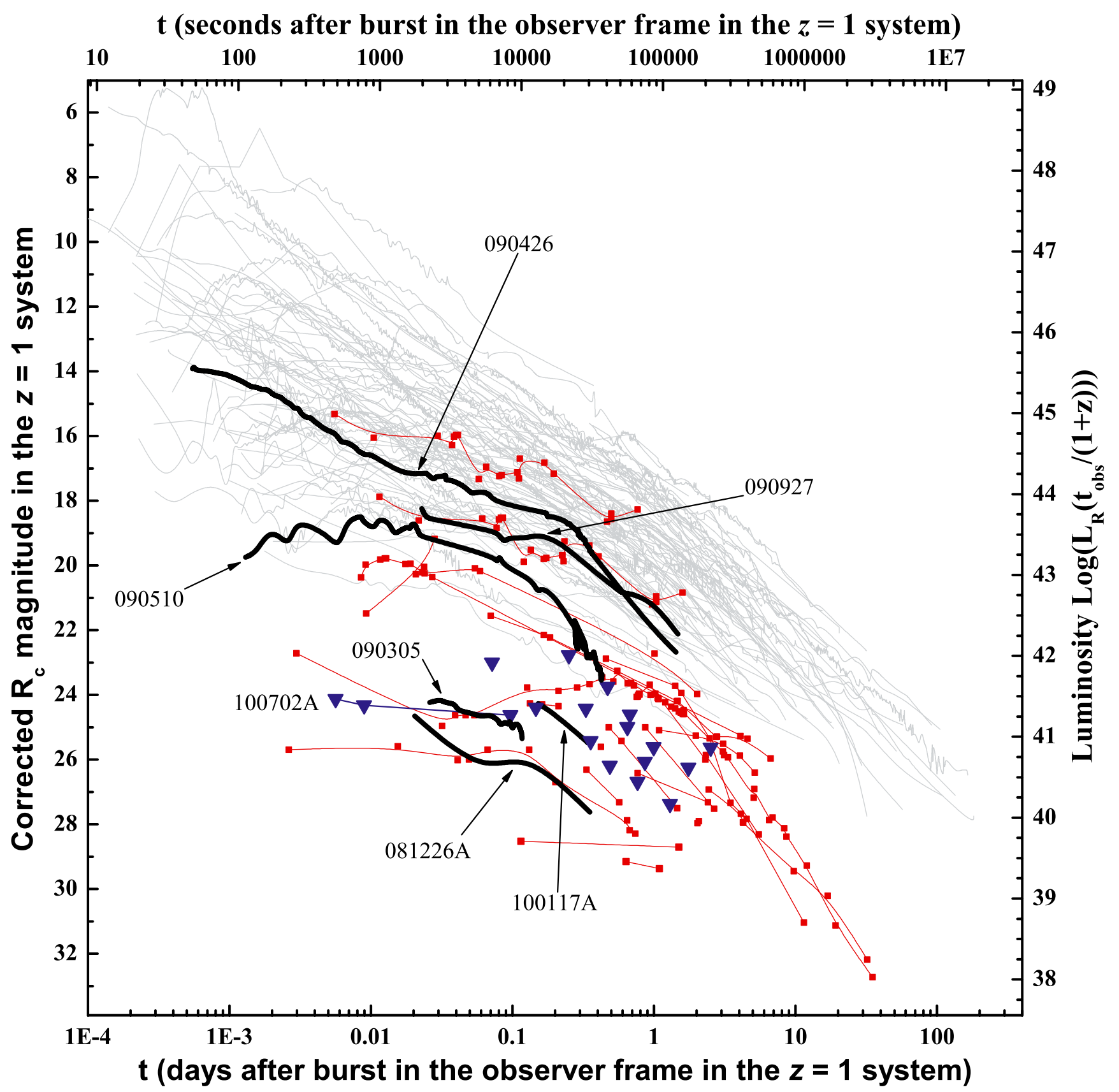

Fig. 15. Light curves of the six GROND-detected short GRB afterglows as well as the upper limits in the redshift $z=1$ frame. The labeling is identical to Fig. 14. GRBs 090426 and 090927 are likely Type II. The luminosity is in units of erg/s. See text for further details.

assumption of $n=0.01 \mathrm{~cm}^{-3}$ for short bursts is also a simplification. For individual bursts it can be wrong by a factor of up to 100 in both directions. Finally, our plot contains only long bursts with measured jet break times. A more detailed study should also contain those long bursts for which only a lower limit on $\Theta_{\text {jet }}$ can be given (e.g., Grupe et al. 2007).

\subsection{X-ray afterglows}

From the Swift Burst Analyser (Evans et al. 2010), we selected all bursts with detected X-ray afterglow and measured redshift that were detected between January 2005 and August 2011 ${ }^{7}$.

\footnotetext{
7 For a more comprehensive statistical analysis of 650 Swift/XRT light curves, we refer to a recent work by Margutti et al. (2012).
}

We then shifted all light curves to their rest frames following Greiner et al. (2009). If no redshift information was available for a short-burst in our sample (Table 1), we assumed a redshift of $z=0.5$.

Figure 17 displays the resulting luminosity evolution of those 14 bursts in our sample for which an X-ray afterglow light curve can be constructed, i.e., the X-ray afterglow is detected during at least two epochs. This excludes GRBs 071112B, 081226B, 090305, 091117A, and 101129A from the plot, which have no afterglow detection at all as well as GRB 100206A, which is only detected once. The figure also shows the luminosity evolution of 191 long GRBs with measured redshift. In addition, we overplot the short-burst sample compiled by Kann et al. (2011), consisting of an additional group of 19 events that are not included in our short-burst sample. 


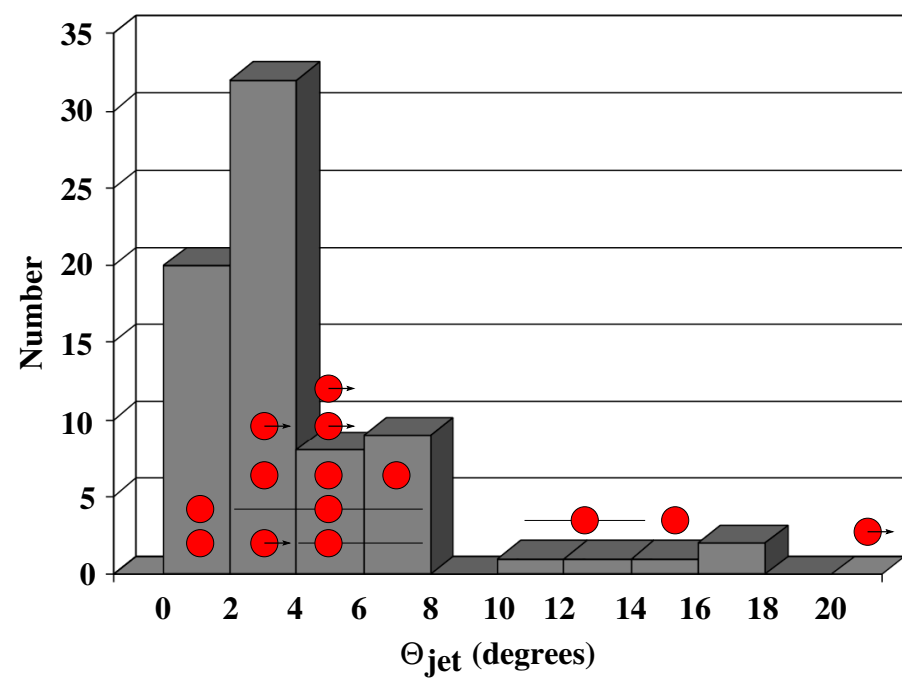

Fig. 16. Observed distribution of jet half-opening angles of 74 long bursts (based on the compilation in $\mathrm{Lu}$ et al. 2012) compared to the short-burst sample. Since the latter has much less data, we do not plot a histogram but only points. An arrow indicates a lower limit on $\Theta_{\text {jet }}$. The Type I events GRB 051221A, 060614, and 070714B listed in Lu et al. (2012) have not been used for the plot of the long-burst data.

Figure 17 demonstrates that the X-ray afterglows of short bursts represent the low end of the luminosity distribution of $\mathrm{X}$-ray afterglows. They are on average a factor of $\sim 100$ less luminous than those of long bursts, similar to what is seen for optical afterglows (Fig. 15; see also Gehrels et al. 2008; Nysewander et al. 2009; Kann et al. 2011). However, with the single exception of GRB 050509B, short bursts do not represent the least luminous X-ray afterglows known. There is a continuous overlap between both populations; for certain time intervals, several long-burst afterglows are even less luminous than the population of short-burst afterglows.

There is a remarkable concentration of short-burst afterglows in a relatively narrow luminosity band around $L_{\mathrm{X},(0.3-10) \mathrm{keV}]} \simeq$ $10^{48} \mathrm{erg} / \mathrm{s}$ at $t \sim 100 \mathrm{~s}$ in the rest frame. Even after removing bursts with assumed redshifts, the concentration is still present, indicating that this is a genuine feature and not an artifact caused by bursts with assumed redshifts. After that time, the luminosities of most short-burst afterglows drop notably and their luminosity distribution broadens by an additional factor of $\sim 10$ to a final range of $\sim 100$, which holds up to at least $t=1 \mathrm{~d}$. At even later times, most short bursts are not detected anymore. Outstanding here is the X-ray afterglow of GRB 060614, which was detected until $t=2 \times 10^{6} \mathrm{~s}$ (rest frame), while in our sample only three events (GRBs 090426, 090927, and 100628A) have been detected beyond $t=10^{5} \mathrm{~s}$. We caution that the former two are possibly Type II GRBs, i.e., originating from the gravitational collapse of a massive star.

In our sample, the X-ray afterglows of the short-bursts GRBs 071227 ( $z=0.383$; D'Avanzo et al. 2007) and 080905A $(z=0.122$; Rowlinson et al. 2010) have the lowest luminosities, while GRB 090927 ( $z=1.37$; Levan et al. 2009a) and $090426(z=2.609$; Levesque et al. 2009) are the most luminous short-bursts in our sample. Again we stress that the latter two are likely Type II GRBs. Adding the data set discussed in Kann et al. (2011), the X-ray afterglow of GRB 050509B represents the low end of the luminosity distribution between $\sim 0.3 \mathrm{ks}$ and $\sim 30 \mathrm{ks}$, followed by GRBs 061201, 060505, and 060614 ${ }^{8}$ at later times. On the other hand, the most luminous short-burst afterglows are those of GRBs 080503 and 051210 , which reach $\left.\log \left(L_{\mathrm{X},(0.3-10) \mathrm{keV}]}[\mathrm{erg} / \mathrm{s}]\right)\right) \simeq 49.25$ during the peak of their emission at $\sim 100$ s. Only the X-ray afterglow of GRB 060121 is more luminous at later times, assuming $z=4.6$ (de Ugarte Postigo et al. 2006); but this GRB is possibly also Type II GRB (Kann et al. 2011).

\section{Summary}

We have reported on the results of 3.5 yrs follow-up observations of short-duration GRBs (defined by $T_{90}<2 \mathrm{~s}$ ) using the multi-channel imager GROND mounted at the 2.2-m telescope on La Silla. GROND is especially designed to perform rapid follow-up observations of afterglows, which is particularly useful for short-duration GRBs because of their on average very faint optical afterglows (Nysewander et al. 2009; Kann et al. 2010, 2011). To our knowledge, what we have presented here is one of the most comprehensive data sets on short-burst followup observations published so far.

In six of the 20 events GROND followed up, it could image the fading optical afterglow. Five of these cases were already known in the literature (GRBs 090305, 090426, 090510, 090927, 100117A), and the GROND follow-up observations of GRBs 090426 and 090510 were already represented in Papers I and II. The new discovery reported here is the optical afterglow of GRB 081226A. It was imaged by GROND superimposed on its faint host galaxy $\left(r^{\prime} \sim 25.8\right)$ and faded away already within $10 \mathrm{ks}$ after the burst. GRB 081226A also belongs to those three cases in our sample where GROND was on target within $10 \mathrm{~min}$ after the trigger. The other two events (GRBs 080919 and $100702 \mathrm{~A}$ ), even though with very small X-ray error circles, were unfortunately located in fields crowded by stars, preventing the discovery of the optical/NIR afterglow in any band.

Three of the six optical afterglow light curves (GRBs 090305, 090426, 090510) show a break that can be interpreted as a jet break. The other three afterglows (GRBs 081226A, 090927, 100117A) show a decay slope in agreement with a pre-jet break evolution, allowing us to set lower constraints on their corresponding jet half-opening angle, $\Theta_{\text {jet }}$. When comparing these results with the long-burst population, we find tentative evidence for wider jet-opening angles of short bursts compared to their long-duration relatives. However, in order to constrain theoretical models, more data are required. Moreover, it should be kept in mind that some long-duration GRBs have relatively large jet-opening angles, too (e.g., Grupe et al. 2007; Racusin et al. 2009; Liang et al. 2008); a clear separation between long and short bursts with respect to their $\Theta_{\text {jet }}$ values obviously does not exist.

The separation between merger and collapsar events becomes more evident when the luminosities of their optical and $\mathrm{X}$-ray afterglows are compared. If the Type I/II classification scheme is used, GRBs 090426 and 090927 have a collapsar origin (Kann et al. 2011), and their afterglow luminosities in the optical band lie in the region occupied by the main body of the long-burst/collapsar population (Fig. 15). The optical luminosities of the afterglows of the Type I GRBs 081226A, 090305, and $100117 \mathrm{~A}$ are substantially smaller and stand apart from the parameter space occupied by the long-burst sample. On the other hand, the optical afterglow of GRB 090510, which was special

8 This is likely a Type I GRB, despite its long duration (Zhang et al. 2009; Kann et al. 2011). 


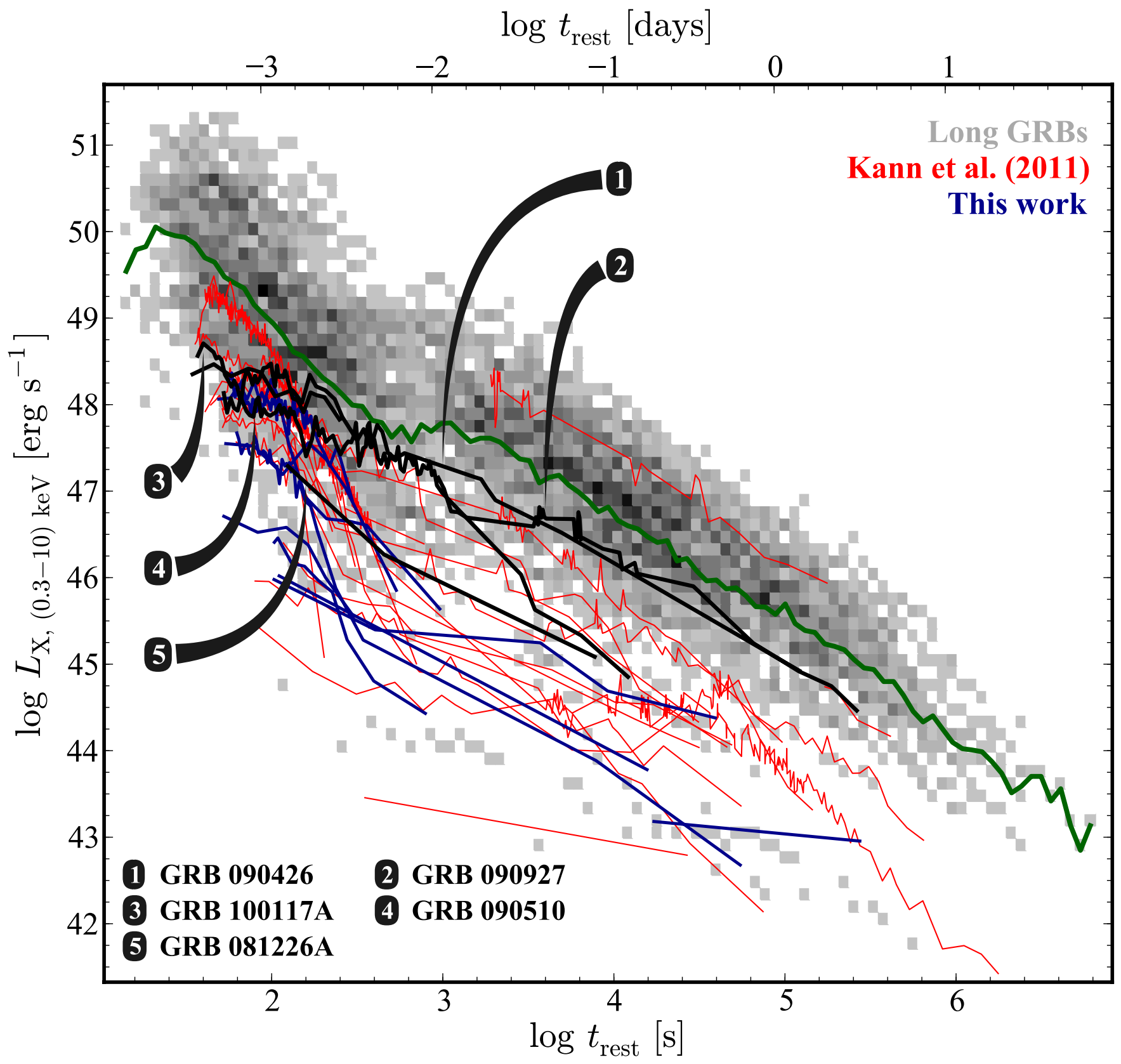

Fig. 17. Luminosity evolution of the X-ray afterglows of the short bursts in our sample. Highlighted are the bursts with optical afterglows (Figs. 14, 15). Black lines represent the afterglows of the likely Type II events GRB 090426 and GRB 00927, green the afterglows of the Type I events GRBs 081226A, 090510, and 100117A. No X-ray afterglow light curve was reported for GRB 090305. Overplotted is also the short-burst sample compiled by Kann et al. (2011) (red color) as well as the X-ray afterglows of the long-burst sample with known redshift (gray). All short-burst afterglows are less luminous than the mean of long-burst afterglow luminosities (orange line); however, there is a continuous overlap between short and long GRB afterglows.

due to its very high-energy emission (see appendix), seems to be an intermediate case.

Seven years after the first precise localizations of shortduration GRBs by Swift, the discovery of their optical afterglows remains an observational challenge. Even though the list of welllocalized short bursts is not that small anymore (Nysewander et al. 2009; Kann et al. 2011; for a continuous update, see footnote 1), the number of well-observed light curves of short burst afterglows is small. Progress in this respect might be strongly linked to the availability of GRB-dedicated instruments on at least medium-class optical telescopes. GROND is one of them.
Acknowledgements. A.N.G., D.A.K., A.R., and S.K. acknowledge support by grant DFG K1 766/16-1. A.N.G., A.R., D.A.K., and A.U. are grateful for travel funding support through the MPE. A.R. acknowledges additional support by the Jenaer Graduiertenakademie. S.S. acknowledges support by a Grant of Excellence from the Iceland Research. T.K. acknowledges funding by the DFG cluster of excellence "Origin and Structure of the Universe", F.O.E. funding of his Ph.D. through the DAAD, M.N. support by DFG grant SA 2001/2-1 and P.S. by DFG grant SA 2001/1-1. Part of the funding for GROND (both hardware and personnel) was generously granted by the Leibniz-Prize to G. Hasinger (DFG grant HA 1850/28-1). This work made use of data supplied by the UK Swift science data center at the University of Leicester. We thank W. Fong and R. Margutti for valuable comments to the manuscript. We thank the referee for a fast and constructive reply. 


\section{References}

Abazajian, K. N., Adelman-McCarthy, J. K., Agüeros, M. A., et al. 2009, ApJS, 182,543

Afonso, P., Krühler, T., Klose, S., \& Greiner, J. 2008, GCN, 8731

Aloy, M. A., Janka, H.-T., \& Müller, E. 2005, A\&A, 436, 273

Andreev, M., Sergeev, A., Parakhin, N., et al. 2010, GCN, 10455

Baumgartner, W. H., Barthelmy, S. D., Cummings, J. R., et al. 2008, GCN, 8275

Baumgartner, W. H., Barthelmy, S. D., Cummings, J. R., et al. 2010, GCN, 10926

Beardmore, A. P., Page, K. L., \& Sakamoto, T. 2007, GCN, 7153

Beardmore, A. P., Cummings, J. R., Holland, S. T., et al. 2009a, GCN, 8932

Beardmore, A. P., Page, K. L., Evans, P. A., et al. 2009b, GRB Coordinates Network, 8937, 1

Beardmore, A. P., Page, K. L., Evans, P. A., et al. 2009c, GCN, 8937

Beckmann, V., Beck, M., Ferrigno, C., et al. 2010, GCN, 10898

Berger, E. 2009, ApJ, 690, 231

Berger, E. 2010a, ApJ, 722, 1946

Berger, E. 2010b, ApJ, 722, 1946

Berger, E. 2010c, GCN, 10908

Berger, E. 2010d, GCN, 10943

Berger, E. 2011, New Astron. Rev., 55, 1

Berger, E., \& Chornock, R. 2010, GCN, 10410

Berger, E., \& Kaplan, D. L. 2007, GCN, 6680

Berger, E., \& Kelson, D. 2009, GCN, 8934

Berger, E., \& Murphy, D. 2007, GCN, 6686

Berger, E., Morrell, N., \& Roth, M. 2007a, GCN, 7151

Berger, E., Morrell, N., \& Roth, M. 2007b, GCN, 7154

Berger, E., Cucchiara, A., \& Fox, D. B. 2008a, GCN, 8732

Berger, E., Soderberg, A. M., Cucchiara, A., \& Fox, D. B. 2008b, GCN, 8736

Berger, E., Chornock, R., Fong, W., Krzeminski, W., \& Anglada, G. 2010a, GCN, 10897

Berger, E., Chornock, R., Tanvir, N., et al. 2010b, GCN, 10395

Berger, E., Fong, W., \& Servillat, M. 2010c, GCN, 10921

Berger, E., Guver, T., \& Fong, W. 2010d, GCN, 10911

Berger, E., Guver, T., Fong, W., \& Chornock, R. 2010e, GCN, 10902

Bhattacharya, D., Mohan, V., Ramaprakash, A. N., Rajarshi, C., \& Misra, K. 2010, GCN, 10380

Bissaldi, E., McBreen, S., Connaughton, V., \& von Kienlin, A. 2008, GCN, 8204

Bloom, J. S., Kulkarni, S. R., \& Djorgovski, S. G. 2002, AJ, 123, 1111

Bloom, J. S., Frail, D. A., \& Kulkarni, S. R. 2003, ApJ, 594, 674

Bloom, J. S., Prochaska, J. X., Pooley, D., et al. 2006, ApJ, 638, 354

Burenin, R., Khorunzhev, G., Sazonov, S., et al. 2010, GCN, 10900

Burrows, D. N., Grupe, D., Capalbi, M., et al. 2006, ApJ, 653, 468

Butler, N. R., Kocevski, D., Bloom, J. S., \& Curtis, J. L. 2007, ApJ, 671, 656

Cano, Z., Melandri, A., Mundell, C., et al. 2009, GCN, 9960

Cenko, S. B., Cobb, B. E., Perley, D. A., \& Bloom, J. S. 2009, GCN, 8933

Cenko, S. B., Bloom, J. S., Perley, D. A., et al. 2010a, GCN, 10389

Cenko, S. B., Perley, D. A., Bloom, J. S., Morgan, A. N., \& Cucchiara, A. 2010b, GCN, 10946

Chandra, P., \& Frail, D. A. 2007, GCN, 6742

Chevalier, R. A., \& Li, Z.-Y. 2000, ApJ, 536, 195

Chornock, R., \& Berger, E. 2011, GCN, 11518

Chornock, R., Fong, W., Berger, E., \& Persson, E. 2010, GCN, 11469

Covino, S., Antonelli, L. A., Calzoletti, L., et al. 2008, GCN, 8271

Cucchiara, A., \& Sakamoto, T. 2007, GCN, 7150

Cummings, J., Barthelmy, S. D., Baumgartner, W., et al. 2008, GCN, 8187

Cummings, J. R., Pal'Shin, V., Yamaoka, K., \& Evans, P. 2009, GCN, 10171

Cummings, J. R., Palmer, D. M., Barthelmy, S. D., et al. 2010, GCN, 11436

D’Avanzo, P., Fiore, F., Piranomonte, S., et al. 2007, GCN, 7152

D’Avanzo, P., Covino, S., Fugazza, D., et al. 2008, GCN, 7157

D'Avanzo, P., Malesani, D., Covino, S., et al. 2009, A\&A, 498, 711

de Pasquale, M., Barthelmy, S. D., Burrows, D. N., et al. 2010a, GCN, 10336

de Pasquale, M., Holland, S. T., \& Oates, S. 2010b, GCN, 10344

de Pasquale, M., Markwardt, C., \& Sbarufatti, B. 2010c, GCN Rep., 269, 1

de Pasquale, M., Schady, P., Kuin, N. P. M., et al. 2010d, ApJ, 709, L146

de Ugarte Postigo, A., Castro-Tirado, A. J., Guziy, S., et al. 2006, ApJ, 648, L83

de Ugarte Postigo, A., Malesani, D., Levan, A. J., Hjorth, J., \& Tanvir, N. R. 2008, GCN, 8195

D'Elia, V., Stratta, G., Perri, M., \& Cummings, J. 2009, GCN, 10292

Della Valle, M., Chincarini, G., Panagia, N., et al. 2006, Nature, 444, 1050

Evans, P. A. 2011a, GCN, 12250

Evans, P. A. 2011b, GCN, 12273

Evans, P. A., Osborne, J. P., \& Goad, M. R. 2008, GCN, 8203

Evans, P. A., Goad, M. R., Osborne, J. P., \& Beardmore, A. P. 2009, GCN, 9950

Evans, P. A., Willingale, R., Osborne, J. P., et al. 2010, A\&A, 519, A102

Fan, Y.-Z., \& Wei, D.-M. 2011, ApJ, 739, 47

Ferrero, P., Kann, D. A., Zeh, A., et al. 2006, A\&A, 457, 857
Flewelling, H., Zheng, W., Pandey, S. B., \& Rujopakarn, W. 2010, GCN, 10917

Fong, W., Berger, E., \& Servillat, M. 2010, GCN, 10919

Fong, W., Berger, E., Chornock, R., et al. 2011, ApJ, 730, 26

Fong, W.-F., Berger, E., Margutti, R., et al. 2012, ApJ, 756, 189

Fox, D. B., Frail, D. A., Price, P. A., et al. 2005, Nature, 437, 845

Frail, D. A., Kulkarni, S. R., Sari, R., et al. 2001, ApJ, 562, L55

Fruchter, A. S., Levan, A. J., Strolger, L., et al. 2006, Nature, 441, 463

Fynbo, J. P. U., Watson, D., Thöne, C. C., et al. 2006, Nature, 444, 1047

Gal-Yam, A., Fox, D. B., Price, P. A., et al. 2006, Nature, 444, 1053

Gehrels, N., Sarazin, C. L., O'Brien, P. T., et al. 2005, Nature, 437, 851

Gehrels, N., Norris, J. P., Barthelmy, S. D., et al. 2006, Nature, 444, 1044

Gehrels, N., Barthelmy, S. D., Burrows, D. N., et al. 2008, ApJ, 689, 1161

Gehrels, N., Ramirez-Ruiz, E., \& Fox, D. B. 2009, ARA\&A, 47, 567

Gelbord, J. M., Barthelmy, S. D., Chester, M. M., et al. 2010, GCN, 11461

Goad, M. R., Osborne, J. P., Beardmore, A. P., \& Evans, P. A. 2010a, GCN, 10378

Goad, M. R., Osborne, J. P., Beardmore, A. P., \& Evans, P. A. 2010b, GCN, 10886

Godet, O. 2008, GCN, 8738

Godet, O., Baumgartner, W. H., Burrows, D. N., et al. 2008, GCN, 8729

Golenetskii, S., Aptekar, R., Mazets, E., et al. 2007a, GCN, 7155

Golenetskii, S., Aptekar, R., Mazets, E., et al. 2007b, GCN, 6690

Golenetskii, S., Aptekar, R., Frederiks, D., et al. 2010, GCN, 11470

Greiner, J., Bornemann, W., Clemens, C., et al. 2007, The Messenger, 130, 12

Greiner, J., Bornemann, W., Clemens, C., et al. 2008, PASP, 120, 405

Greiner, J., Krühler, T., Fynbo, J. P. U., et al. 2009, ApJ, 693, 1610

Gronwall, C., \& Grupe, D. 2009, GCN, 9946

Gruber, D., Bissaldi, E., \& McBreen, S. 2009, GCN, 9974

Grupe, D., \& Siegel, M. 2010, GCN, 10924

Grupe, D., Burrows, D. N., Patel, S. K., et al. 2006, ApJ, 653, 462

Grupe, D., Gronwall, C., Wang, X.-Y., et al. 2007, ApJ, 662, 443

Grupe, D., Cummings, J. R., Gronwall, C., et al. 2009a, GCN, 9945

Grupe, D., Kuin, P., Cummings, J. R., et al. 2009b, GCN Rep., 252

Guidorzi, C., Barbier, L. M., Barthelmy, S. D., et al. 2007a, GCN, 6678

Guidorzi, C., Romano, P., \& Moretti, A. 2007b, GCN, 6682

Guidorzi, C., Sato, G., Cucciara, A., et al. 2007c, GCN Rep., 77

Guziy, S., Aceituno, F. J., \& Castro-Tirado, A. J. 2010, GCN, 10384

He, H.-N., Wu, X.-F., Toma, K., Wang, X.-Y., \& Mészáros, P. 2011, ApJ, 733, 22

Hjorth, J., Sollerman, J., Møller, P., et al. 2003, Nature, 423, 847

Hjorth, J., Sollerman, J., Gorosabel, J., et al. 2005, ApJ, 630, L117

Holland, S. T., Burrows, D. N., Kennea, J. A., et al. 2010a, GCN, 10884

Holland, S. T., Landsman, W. B., Page, K. L., \& Stamatikos, M. 2010b, GCN Rep., 289

Hoversten, E. A., \& Godet, O. 2008, GCN, 8737

Hoversten, E. A., Krimm, H. A., Grupe, D., et al. 2009, GCN Rep., 218

Immler, S. 2010, GCN, 10901

Immler, S., \& Holland, S. T. 2008, GCN, 8277

Immler, S., Starling, R. L. C., Evans, P. A., Barthelmy, S. D., \& Sakamoto, T. 2010, GCN Rep., 290

Kann, D. A., Klose, S., \& Zeh, A. 2006, ApJ, 641, 993

Kann, D. A., Klose, S., Zhang, B., et al. 2010, ApJ, 720, 1513

Kann, D. A., Klose, S., Zhang, B., et al. 2011, ApJ, 734, 96

Kawabata, K. S., Deng, J., Wang, L., et al. 2003, ApJ, 593, L19

Klotz, A., Vaalsta, T. P., Yan, L., et al. 2009, GCN, 9956

Kouveliotou, C., \& Connaughton, V. 2009, GCN, 8785

Kouveliotou, C., Meegan, C. A., Fishman, G. J., et al. 1993, ApJ, 413, L101

Krimm, H. A., Barthelmy, S. D., Baumgartner, W. H., et al. 2008, GCN, 8735

Krimm, H. A., Barthelmy, S. D., Beardmore, A. P., et al. 2009, GCN, 8936

Krimm, H. A., Barthelmy, S. D., Baumgartner, W. H., et al. 2010a, GCN, 11467

Krimm, H. A., Baumgartner, W. H., Evans, P. A., et al. 2010b, GCN, 10376

Krimm, H. A., Cummings, J. R., Evans, P. A., \& Marshall, F. E. 2010c, GCN Rep., 271

Krühler, T., Küpcü Yoldaş, A., Greiner, J., et al. 2008, ApJ, 685, 376

Kuin, N. P. M., \& Grupe, D. 2009, GCN, 9954

Kumar, P., \& Barniol Duran, R. 2010, MNRAS, 409, 226

Küpcü Yoldaş, A., Krühler, T., Greiner, J., et al. 2008, in AIP Conf. Ser. 1000, ed. M. Galassi, D. Palmer, \& E. Fenimore, 227

Leibler, C. N., \& Berger, E. 2010, ApJ, 725, 1202

Leloudas, G., Xu, D., Malesani, D., et al. 2010, GCN, 10387

Levan, A. J., \& Tanvir, N. R. 2010, GCN, 10887

Levan, A. J., Fynbo, J. P. U., Hjorth, J., et al. 2009a, GCN, 9958

Levan, A. J., Tanvir, N. R., Hjorth, J., et al. 2009b, GCN, 10154

Levan, A. J., Graham, J., Fruchter, A., et al. 2010a, GCN, 10349

Levan, A. J., Tanvir, N. R., Wiersema, K., \& Cenko, S. B. 2010b, GCN, 10909

Levan, A. J., Tanvir, N. R., Wiersema, K., et al. 2010c, GCN, 10386

Levesque, E., Chornock, R., Kewley, L., et al. 2009, GCN, 9264 
Liang, E., Racusin, J. L., Zhang, B., Zhang, B., \& Burrows, D. N. 2008, ApJ, 675,528

Lu, R.-J., Wei, J.-J., Qin, S.-F., \& Liang, E.-W. 2012, ApJ, 745, 168

MacFadyen, A. I., \& Woosley, S. E. 1999, ApJ, 524, 262

Malesani, D., Covino, S., D'Avanzo, P., et al. 2007, A\&A, 473, 77

Malesani, D., de Ugarte Postigo, A., Fynbo, J. P. U., et al. 2008, GCN, 8190

Malesani, D., de Ugarte Postigo, A., Levan, A. J., et al. 2009, GCN, 10156

Malesani, D., Levan, A. J., \& Fynbo, J. P. U. 2010, GCN, 10918

Mao, J., Wang, D., \& Bai, J. 2010, GCN, 10392

Margutti, R., Zaninoni, E., Bernardini, M. G., Chincarini, G., \& for the SwiftXRT team 2012 [arXiv: 1207.0537]

Markwardt, C. B., Barthelmy, S. D., Baumgartner, W. H., et al. 2010, GCN, 10338

Marshall, F. E., \& Krimm, H. A. 2010, GCN, 10394

Matheson, T., Garnavich, P. M., Stanek, K. Z., et al. 2003, ApJ, 599, 394

Mazzali, P. A., Deng, J., Nomoto, K., et al. 2006, Nature, 442, 1018

McBreen, S., Krühler, T., Rau, A., et al. 2010, A\&A, 516, A71

Mereghetti, S., Paizis, A., Gotz, D., et al. 2008, GCN, 8734

Miller, A. A., Perley, D. A., Bloom, J. S., Cenko, S. B., \& Nugent, P. E. 2010, GCN, 10377

Moin, A., Frail, D. A., Tingay, S., \& Macquart, J. 2009a, GCN, 10021

Moin, A., Tingay, S., Phillips, C., et al. 2009b, GCN, 8952

Morgan, A. N., Cobb, B. E., Bloom, J. S., et al. 2010, GCN, 10390

Nakar, E. 2007, Phys. Rep., 442, 166

Nardini, M., Ghisellini, G., Ghirlanda, G., et al. 2006, A\&A, 451, 821

Nicuesa Guelbenzu, A., Krühler, T., Klose, S., \& Greiner, J. 2010, GCN, 10396

Nicuesa Guelbenzu, A., Klose, S., Rossi, A., et al. 2011, A\&A, 531, L6 (Paper I)

Nicuesa Guelbenzu, A., Klose, S., Krühler, T., et al. 2012, A\&A, 538, L7 (Paper II)

Noda, K., Sonoda, E., Hayasi, H., et al. 2010, GCN, 10385

Nysewander, M., Fruchter, A. S., \& Pe'er, A. 2009, ApJ, 701, 824

Oates, S. R., Barthelmy, S. D., Baumgartner, W. H., et al. 2009a, GCN, 10148

Oates, S. R., Page, K. L., Evans, P. A., \& Markwardt, C. B. 2009b, GCN Rep., 259

Onda, K., Tashiro, M., Terada, Y., et al. 2008, GCN, 7158

Paciesas, W. 2010, GCN, 10345

Paczyński, B. 1986, ApJ, 308, L43

Pagani, C., Baumgartner, W. H., Beardmore, A. P., et al. 2008a, GCN, 8180

Pagani, C., Racusin, J. L., Brown, P., \& Cummings, J. 2008b, GCN Rep., 162

Panaitescu, A. 2011, MNRAS, 414, 1379

Panaitescu, A., \& Kumar, P. 2001, ApJ, 554, 667

Panaitescu, A., Kumar, P., \& Narayan, R. 2001, ApJ, 561, L171

Perley, D. A., Cenko, S. B., Bloom, J. S., et al. 2009, AJ, 138, 1690

Perley, D. A., Berger, E., Cenko, S. B., et al. 2010, GCN, 11464

Perley, D. A., Modjaz, M., Morgan, A. N., et al. 2011, ApJ, in press [arXiv: 1112.3963]
Perri, M., Stratta, G., Fenimore, E., et al. 2007, GCN Rep., 103 Pian, E., Mazzali, P. A., Masetti, N., et al. 2006, Nature, 442, 1011 Preger, B., Barthelmy, S. D., Baumgartner, W. H., et al. 2008a, GCN, 8270 Preger, B., Perri, M., Stratta, G., et al. 2008b, GCN Rep., 172 Preger, B., Stratta, G., \& Perri, M. 2008c, GCN, 8276

Racusin, J. L., Liang, E. W., Burrows, D. N., et al. 2009, ApJ, 698, 43 Rau, A., McBreen, S., \& Krühler, T. 2009, GCN, 9353 Rezzolla, L., Giacomazzo, B., Baiotti, L., et al. 2011, ApJ, 732, L6 Rowlinson, A., Wiersema, K., Levan, A. J., et al. 2010, MNRAS, 408, 383 Rumyantsev, V., Shakhovkoy, D., \& Pozanenko, A. 2010, GCN, 10456 Sakamoto, T., Baumgartner, W. H., Beardmore, A. P., et al. 2007a, GCN, 7147 Sakamoto, T., Norris, J., Ukwatta, T., et al. 2007b, GCN, 7156

Sakamoto, T., Barthelmy, S. D., Baumgartner, W. H., et al. 2009, GCN, 10180 Sakamoto, T., Barthelmy, S. D., Baumgartner, W. H., et al. 2010, GCN, 10379 Sakamoto, T., Barthelmy, S. D., Baumgartner, W. H., et al. 2011, ApJS, 195, 2 Sari, R., Piran, T., \& Halpern, J. P. 1999, ApJ, 519, L17

Sato, G., Barbier, L., Barthelmy, S. D., et al. 2007, GCN, 7148

Sato, G., Barthelmy, S. D., Baumgartner, W. H., et al. 2009, GCN, 9263

Sbarufatti, B., Margutti, R., \& de Pasquale, M. 2010, GCN, 10342

Schaefer, B. E., Rykoff, E. S., \& Yuan, F. 2008, GCN, 8730

Schlegel, D. J., Finkbeiner, D. P., \& Davis, M. 1998, ApJ, 500, 525

Schulze, S., Klose, S., Björnsson, G., et al. 2011, A\&A, 526, A23

Siegel, M. H., Barthelmy, S. D., Burrows, D. N., et al. 2010a, GCN, 10916

Siegel, M. H., Grupe, D., de Pasquale, M., et al. 2010b, GCN Rep., 292

Skrutskie, M. F., Cutri, R. M., Stiening, R., et al. 2006, AJ, 131, 1163

Soderberg, A. M., Berger, E., Kasliwal, M., et al. 2006, ApJ, 650, 261

Spergel, D. N., Verde, L., Peiris, H. V., et al. 2003, ApJS, 148, 175

Stamatikos, M., Barthelmy, S. D., Baumgartner, W. H., et al. 2009, GCN, 9955

Stanek, K. Z., Matheson, T., Garnavich, P. M., et al. 2003, ApJ, 591, L17

Starling, R. L. C., \& Immler, S. 2010, GCN, 10899

Starling, R. L. C., Evans, P. A., \& Immler, S. 2010, GCN, 10941

Stratta, G., D’ Avanzo, P., Piranomonte, S., et al. 2007, A\&A, 474, 827

Suzuki, D., Omori, K., Hayashi, F., et al. 2010, GCN, 10904

Tanvir, N. R., \& Levan, A. J. 2010, GCN, 10905

Thöne, C. C., Fynbo, J. P. U., Östlin, G., et al. 2008, ApJ, 676, 1151

Thöne, C. C., Campana, S., Lazzati, D., et al. 2011, MNRAS, 414, 479

Tody, D. 1993, ASP Conf. Ser., 52, 173

Usov, V. V. 1992, Nature, 357, 472

von Kienlin, A. 2010, GCN, 10381

Woosley, S. E., \& Bloom, J. S. 2006, ARA\&A, 44, 507

Yurkov, V., Sergienko, S., Gorbovskoy, E., et al. 2010, GCN, 10391

Zeh, A., Klose, S., \& Hartmann, D. H. 2004, ApJ, 609, 952

Zeh, A., Klose, S., \& Kann, D. A. 2006, ApJ, 637, 889

Zhang, W., Woosley, S. E., \& Heger, A. 2004, ApJ, 608, 365

Zhang, B., Zhang, B.-B., Liang, E.-W., et al. 2007, ApJ, 655, L25

Zhang, B., Zhang, B.-B., Virgili, F. J., et al. 2009, ApJ, 703, 1696 


\section{Appendix A: Data tables}

Table A.1. Log of the GROND observations of the afterglow (plus host) of GRB 081226A (Fig. 2).

\begin{tabular}{lccccccc}
\hline \hline Time (s) & $g^{\prime}$ & $r^{\prime}$ & $i^{\prime}$ & $z^{\prime}$ & $J$ & $H$ & $K_{\mathrm{s}}$ \\
\hline 1320 & $>24.1$ & $23.59(22)$ & $>23.1$ & $22.86(35)$ & $>20.9$ & $>20.3>19.6$ \\
4070 & $25.48(30)$ & $24.76(24)$ & $24.40(35)$ & $23.73(24)$ & $>21.8$ & $>21.3>20.1$ \\
21650 & $25.56(23)$ & $25.75(34)$ & $>24.9$ & $>24.5$ & $>21.9>21.4>20.3$ \\
$2.44 \mathrm{E} 6$ & $25.85(24)$ & $25.75(34)$ & $>25.0$ & $>24.5$ & $>22.2$ & $>21.6>20.6$ \\
\hline
\end{tabular}

Notes. These results supercede the data given in Afonso et al. (2008).

Table A.2. Log of the GROND observations of the afterglow of GRB 090305 (Fig. 4).

\begin{tabular}{lcccc}
\hline \hline Time $(\mathrm{s})$ & $g^{\prime}$ & \multicolumn{1}{c}{$r^{\prime}$} & $i^{\prime}$ & $z^{\prime}$ \\
\hline 2014 & - & $23.13(09)$ & $22.96(18)$ & - \\
2568 & $23.77(19)$ & $23.26(13)$ & - & - \\
3318 & $23.79(15)$ & $23.55(15)$ & $23.24(19)$ & - \\
3925 & - & $23.68(13)$ & - & - \\
4367 & - & $23.61(07)$ & - & - \\
4594 & $24.07(09)$ & - & $23.55(13)$ & - \\
4814 & - & $23.92(11)$ & - & - \\
5262 & - & $23.82(20)$ & - & - \\
5495 & - & - & - & $23.46(12)$ \\
5719 & - & $23.74(11)$ & - & - \\
6166 & - & $23.91(08)$ & - & - \\
6392 & $24.534(13)$ & - & $23.70(11)$ & - \\
6613 & - & $23.91(08)$ & - & - \\
7065 & - & $23.86(08)$ & - & - \\
7519 & - & $24.14(27)$ & - & - \\
\hline
\end{tabular}

Table A.3. Log of the Gemini observations of the afterglow of GRB 090305 (Fig. 4).

\begin{tabular}{lccc}
\hline \hline $\begin{array}{l}\text { Mid-time } \\
(\mathrm{s})\end{array}$ & $\begin{array}{c}g^{\prime} \\
\mathrm{mag}\end{array}$ & $\begin{array}{c}\text { Mid-time } \\
(\mathrm{s})\end{array}$ & $\begin{array}{c}r^{\prime} \\
\mathrm{mag}\end{array}$ \\
\hline 2859 & $23.89(05)$ & 1681 & $23.18(03)$ \\
3329 & $23.94(05)$ & 2150 & $23.21(03)$ \\
3800 & $24.02(07)$ & 2621 & $23.43(04)$ \\
- & - & 5220 & $23.77(05)$ \\
- & - & 5689 & $23.82(05)$ \\
- & - & 6159 & $23.89(05)$ \\
- & - & 6478 & $24.04(04)$ \\
- & - & 7587 & $24.29(04)$ \\
\hline
\end{tabular}

Table A.4. $\log$ of the GROND observations of the afterglow of GRB 090927 (Fig. 6).

\begin{tabular}{llc}
\hline \hline Time $(\mathrm{s})$ & \multicolumn{1}{c}{$r^{\prime}$} & $i^{\prime}$ \\
\hline 61700 & $21.90(09)$ & $21.79(06)$ \\
62380 & $21.86(15)$ & - \\
63036 & $21.93(05)$ & - \\
65325 & $21.89(07)$ & - \\
150945 & $23.18(21)$ & $23.03(22)$ \\
\hline
\end{tabular}

\section{Appendix B: GRBs without afterglow detection by GROND}

\section{B.1. GRB 070729}

The original $90 \%$ c.l. XRT error circle radius was 5".7 (Guidorzi et al. 2007a), which was refined to 4.'5 some hours later (Guidorzi et al. 2007b). A host galaxy candidate was soon reported (Berger \& Kaplan 2007). However, the final XRT position lies about $9^{\prime \prime}$ northeast and does not overlap with the previous XRT error circle (Evans 2011a,b).

GRB 070729 was the first short GRB observed with GROND after its commissioning in mid-2007. GROND observations started $6 \mathrm{~h}$ after the burst and continued for $4.5 \mathrm{~h}$ until sunrise. A second-epoch observation was performed the following night for $1 \mathrm{~h}$. No transient object between the two epochs was detected in any band (Küpcü Yoldaş et al. 2008).

\section{B.2. GRB 071227}

GROND started observing the field $4 \mathrm{~h}$ after the GRB trigger. At that time, the weather conditions were not good. GROND could not detect the afterglow in any band (Table 4). Second-epoch observations were performed the following night. GROND was on target $29 \mathrm{~h}$ after the burst and observed for one hour. At that time, the host galaxy had already been discovered by Swift/UVOT (Sakamoto et al. 2007a; Cucchiara \& Sakamoto 2007), and its redshift was measured to be $z=0.381 \pm 0.001$ (D'Avanzo et al. 2007, 2009; Berger et al. 2007b). VLT observations revealed an optical afterglow situated 3'. 1 away from the center of its host, an edge-on galaxy (D'Avanzo et al. 2008, 2009). GROND could not detect the afterglow anymore, only deep limiting magnitudes can be provided: $g^{\prime} r^{\prime} i^{\prime} z^{\prime} J H=25.5,25.0,24.2,24.4,21.5,20.5$ at $29 \mathrm{~h}$ after the burst. The $r^{\prime}$-band upper limit is in agreement with the expectations based on the VLT $R$-band detection at $0.3 \mathrm{~d}$ after the burst if the optical afterglow was fading analogeous to its X-ray counterpart with a decay slope of $\alpha \sim 1$ (see Fig. 7 in D'Avanzo et al. 2009). The GRB host galaxy is discussed in detail by D'Avanzo et al. (2009).

\section{B.3. GRB $080905 A$}

GROND started observing the field of GRB 080905A about $17.5 \mathrm{~h}$ after the burst. Observations continued for only $11 \mathrm{~min}$ at a seeing of $2^{\prime \prime}$. 2 . The combined $g^{\prime} r^{\prime} i^{\prime} z^{\prime}$-band image as well as the combined $J H K_{\mathrm{s}}$-band image do not show the afterglow and faint host galaxy discovered with the ESO/VLT (Rowlinson et al. 2010). Our non-detection is in agreement with these authors, according to whom at the time of our observations the magnitude of the afterglow was around $R_{\mathrm{C}}=24$, about 1 mag below our detection limit. Although the field is very crowded with stars, the afterglow was situated in a region free of stars. In addition, it was well separated from the center of its suspected anonymous host galaxy. Therefore, the upper limits we can provide (Table 4) are not affected by the light of the host galaxy. We refer to Rowlinson et al. (2010) for a detailed study of this burst and its host galaxy.

\section{B.4. GRB 091109B}

GROND observed GRB 091109B six hrs after the trigger. The weather conditions over La Silla observatory were not good at that time. Although GROND was on target for one hour, observations were not deep enough because of clouds. Inside the 
2.'8 90\% c.l. XRT error circle, no source can be detected in the GROND images (Table 4).

A faint optical transient was discovered by VLT/FORS in the $R_{\mathrm{C}}$ band at the time when GROND was observing (Levan et al. 2009b; Malesani et al. 2009), but it was not detected in the NIR (VLT/High Acuity Wide field $K$-band Imager (HAWK-I)). The non-detection of the afterglow by GROND is in agreement with the magnitude reported by Levan et al. (2009b), $R_{\mathrm{C}} \sim 25$, which is deeper than our limiting $r^{\prime}$-band magnitude (23.3; Table 4). Re-analysing the VLT/FORS data, we find that from $20 \mathrm{ks}$ to $40 \mathrm{ks}$ the light curve of the afterglow can be fitted with a single power-law with a slope of $\alpha=0.80 \pm 0.04$. For this time period, there are also simultaneous Swift/XRT observations which, within errors, can be fitted with the same decay slope $\left(\alpha_{\mathrm{X}}=1.08 \pm 0.36\right)$.

\section{B.5. GRB $100206 A$}

GROND started observing the field $11 \mathrm{~h}$ after the trigger. Observations were performed at high airmass and under poor seeing conditions. No evidence for an afterglow candidate was found in any band (Nicuesa Guelbenzu et al. 2010). Perley et al. (2011) published a detailed investigation of the GRB host galaxy.

\section{B.6. GRB $100625 A$}

GROND visited the field of GRB 100625A several times. Firstepoch observations started $11.7 \mathrm{~h}$ after the GRB trigger and lasted for about $1 \mathrm{~h}$. Second-epoch observations were done on June 27, about $39 \mathrm{~h}$ after the trigger, and a third run was performed on July 1 (about $5.5 \mathrm{~d}$ after the trigger). Further data of the field were collected in 2010.

Within the $r=1$ ". $890 \%$ c.l. XRT error circle (Goad et al. 2010b), an object is detected in all GROND epochs, the potential GRB host galaxy (Berger et al. 2010a). No evidence was found in the GROND data for a decaying afterglow superimposed upon this galaxy (Table 4; note that these upper limits refer to an isolated afterglow).

\section{B.7. GRB 100628 A}

GROND started observing the field about $17 \mathrm{~h}$ after the GRB trigger and remained on target for $1.5 \mathrm{~h}$. At that time, two extended objects were already detected inside the final $90 \%$ c.l. XRT error circle (Berger et al. 2010e,d). No optical afterglow was detected.

\section{B.8. GRB 101219A}

Observations with GROND started about 80 min after the GRB trigger and continued for about two hrs. Although they were performed under good weather conditions (seeing 0'. 8 , airmass 1.1), the proximity of the Moon affected the depth of the observations. No optical transient was detected by GROND in any band down to deep flux limits (Table 4).

\section{B.9. GRBs with arcmin-sized error circles}

This sample contains four bursts where only a Swift/BAT or, in one case, an INTEGRAL/IBIS error circle is known. They are typically $3 \mathrm{arcmin}$ in radius. These events are GRBs 071112B, 081226B, 091117A, and 101129A. Because of visibility constraints by GROND or Swift/XRT in these cases, GROND was on target not earlier than between 6 and $31 \mathrm{~h}$ after the corresponding GRB trigger. Given that, on average, short GRB afterglows are intrinsically substantially fainter than those of long GRBs (see Kann et al. 2010, 2011), it was not very likely that in these cases GROND could image the afterglow in any band. Indeed, only upper limits can be provided (Table 4).

\section{Appendix C: Additional observations reported in the literature}

\section{C.1. GRB 070729}

Swift/BAT triggered on GRB 070729 at 00:25:53 UT (Guidorzi et al. 2007a) and had a duration of $T_{90}(15-350 \mathrm{keV})=0.9 \pm 0.1 \mathrm{~s}$ (Guidorzi et al. 2007c). The burst was also seen by Konus $A$ (Golenetskii et al. 2007b). An uncatalogued X-ray source was found by Swift/XRT but no optical afterglow by Swift/UVOT (Guidorzi et al. 2007a). Inside the initial $r=5$ !'7 XRT error circle, Berger \& Kaplan (2007) reported the detection of an extended object visible in the $K$ band. A refined XRT error circle with a radius of $r=4 . .5$ was later reported by Guidorzi et al. (2007b). This error circle lies 3'.2 away from the initial XRT position. Optical follow-up observations were performed in the $R$ band with the Swope 40-inch telescope at Las Campanas Observatory, but no sources were detected inside the XRT error circles, implying that the aforementioned galaxy is a red object (Berger \& Murphy 2007). No afterglow was detected in the radio band (Chandra \& Frail 2007). The position of the XRT afterglow was later refined and shifted by about $5^{\prime \prime}$ in NE direction while it shrunk to $r=2$ '.5 (Evans 2011a,b).

\section{C.2. GRB 071227}

This was a bright and multi-peaked GRB with $T_{90}(15-$ $350 \mathrm{keV})=1.8 \pm 0.4 \mathrm{~s}$ that triggered Swift/BAT at 20:13:47 UT (Sakamoto et al. 2007a; Sato et al. 2007). It was also detected by Konus-Wind (Golenetskii et al. 2007a) and Suzaku/Wide-Band All-Sky Monitor (WAM; Onda et al. 2008). Swift localized a bright X-ray afterglow (Beardmore et al. 2007). UVOT observations (Sakamoto et al. 2007a; Cucchiara \& Sakamoto 2007) revealed a single faint source near the XRT error circle, which was identified as a galaxy also visible in the DSS (Berger et al. 2007a). VLT (D'Avanzo et al. 2007, 2009) and Magellan (Berger et al. 2007b) spectroscopy revealed a redshift of this galaxy of $z=0.381 \pm 0.001$, and further VLT follow-up detected the optical afterglow (D'Avanzo et al. 2008, 2009) at the tip of this edge-on spiral galaxy.

\section{C.3. GRB $080905 A$}

Swift/BAT and Fermi/GBM triggered on GRB 080905 at 11:58:55 UT (Pagani et al. 2008a; Bissaldi et al. 2008). The BAT light curve shows three peaks with a total duration of about $2 \mathrm{~s}$ (Pagani et al. 2008a). Its duration was $T_{90}(15-350 \mathrm{keV})=$ $1.1 \pm 0.1 \mathrm{~s}$ (Cummings et al. 2008). A fading X-ray afterglow was found, but no optical afterglow was detected with UVOT (Pagani et al. 2008a). A faint afterglow candidate was then discovered with the VLT (Malesani et al. 2008), and a host galaxy was also seen (de Ugarte Postigo et al. 2008). The revised $r=11^{\prime \prime} 6$ XRT error circle is in agreement with this afterglow position (Evans et al. 2008). The afterglow is located in an outer arm of a starforming spiral galaxy at $z=0.1218 \pm 0.0003$, making it the 
closest short GRB known so far. This event has been analyzed in detail by Rowlinson et al. (2010).

\section{C.4. GRB 080919}

GRB 080919 triggered Swift/BAT at 00:05:13 UT. The burst consists of a single spike and had a total duration of $T_{90}(15-$ $350 \mathrm{keV})=0.6 \pm 0.1 \mathrm{~s}$. Swift/XRT began observing about $71 \mathrm{~s}$ after the BAT trigger. The detected X-ray afterglow could be localized with high precision ( $r=2$ '. 1; Preger et al. 2008a), but remained undetected from the second orbit on. Swift/UVOT started observing about $11 \mathrm{~s}$ after XRT, but no afterglow candidate could be found in the white filter down to $m=18$ (Preger et al. 2008b; Baumgartner et al. 2008; Immler \& Holland 2008). The size of the X-ray error circle could finally be improved to $r=2$ '.0 (Preger et al. 2008c). Ground-based observations with the robotic REM telescope on La Silla, Chile, started already $74 \mathrm{~s}$ after the BAT trigger and revealed a bright NIR source in the XRT error circle $(H=13.73 \pm 0.03)$. This source, however, is also listed in the 2MASS catalogue and might therefore be an unrelated Galactic foreground object (Covino et al. 2008). No further follow-up observations are reported in the literature. The position of the XRT afterglow was slightly refined three years after the event (Evans 2011a,b).

\section{C.5. GRB 081226A}

GRB 081226A triggered Fermi/Gamma-ray Burst Monitor (GBM) and Swift/BAT at 01:03:37 UT (Godet et al. 2008; Kouveliotou \& Connaughton 2009). Its duration was $T_{90}(15-$ $350 \mathrm{keV})=0.4 \pm 0.1 \mathrm{~s}$ (Krimm et al. 2008). Swift/XRT started observing the field $94.5 \mathrm{~s}$ after the BAT trigger, and an afterglow was found (Godet 2008). UVOT started observing $156 \mathrm{~s}$ after the trigger, but no optical afterglow was identified (Hoversten \& Godet 2008). Optical observations by Robotic Optical Transient Search Experiment (ROTSE)-IIIc starting $25 \mathrm{~s}$ after the GRB could only reveal upper limits on any optical afterglow (Schaefer et al. 2008). GROND detected an afterglow candidate (Afonso et al. 2008), but observations with Gemini-S did not reveal a fading behavior, neither of this source nor of a second one found in the XRT error circle (Berger et al. 2008a,b). No radio counterpart of the optical afterglow candidate(s) could be found with the Australia Telescope Compact Array (ATCA; Moin et al. 2009b). The position of the XRT afterglow was slightly refined three years after the event (Evans 2011a,b).

\section{C.6. GRB 090305}

The burst triggered Swift/BAT at 05:19:51 UT. The BAT light curve shows a single short spike with a duration of $T_{90}(15-$ $350 \mathrm{keV})=0.4 \pm 0.1 \mathrm{~s}$. XRT began observing the field $93 \mathrm{~s}$ after the trigger, but no X-ray afterglow was initially detected. UVOT started observing 96 seconds after the trigger, but no optical afterglow candidate was discovered either (Beardmore et al. 2009a; Krimm et al. 2009). Despite the lack of an XRT position, rapid follow-up of the BAT error circle with Gemini-S/GMOS and Magellan/Baade led to the discovery of the optical afterglow (Cenko et al. 2009; Berger \& Kelson 2009). In addition, a re-analysis with relaxed constraints allowed the detection of an extremely faint X-ray afterglow at the position of the optical counterpart (Beardmore et al. 2009c). No host galaxy was detected down to deep limits right under the optical afterglow position (Berger 2010b).

\section{C.7. GRB 090927}

The burst was detected by Swift/BAT at 10:07:16 UT (Grupe et al. 2009a). It had a Fast Rise Exponential Decay (FRED)like shape with some substructure and a duration of $T_{90}(15-$ $350 \mathrm{keV})=2.2 \pm 0.4 \mathrm{~s}$ (Stamatikos et al. 2009). It was also detected by Fermi/GBM (Gruber et al. 2009). The final classification of the burst is not totally clear. It is more likely a long GRB because it shows significant spectral lag and was relatively soft (Grupe et al. 2009b). After its BAT trigger, Swift could not immediately slew to the field due to an Earth-limb constraint. When Swift/UVOT began observing the field 2121 s after the trigger, it immediately discovered an optical afterglow candidate (Gronwall \& Grupe 2009; Kuin \& Grupe 2009). Only thereafter was the detection of the X-ray afterglow announced, a quite unusual situation (Evans et al. 2009). The afterglow was observed with the 1-m f/4 Zadko telescope in Western Australia (Klotz et al. 2009), which started observations $50 \mathrm{~min}$ after the trigger (with the first magnitude value for $t \sim 2 \mathrm{~h}$ ). It was also observed with the Faulkes Telescope South in Australia (Cano et al. 2009), which observed $4.2 \mathrm{~h}$ after the onset of the GRB, and with VLT/FORS2 on ESO Paranal (Levan et al. 2009a), which observed $16.5 \mathrm{~h}$ after the burst trigger. The VLT observations allowed for a measurement of the afterglow redshift $(z=1.37$; Levan et al. 2009a). Radio observations with the Australian ATCA array did not reveal the afterglow (Moin et al. 2009a).

\section{C.8. GRB 091109B}

Swift/BAT triggered on GRB 091109B at 21:49:03 UT ( $T_{90}(15-$ $350 \mathrm{keV})=0.30 \pm 0.03 \mathrm{~s}$; Oates et al. 2009b). An X-ray afterglow was immediately detected, but no optical afterglow was found (Oates et al. 2009a). The burst was a symmetrical spike with no sign of extended emission (Oates et al. 2009b). A faint, rapidly decaying afterglow was discovered with the VLT at coordinates RA, Dec $(J 2000)=07: 30: 56.61,-54: 05: 22.85$ (Levan et al. 2009b; Malesani et al. 2009).

\section{C.9. GRB 100117A}

The burst triggered Swift/BAT (de Pasquale et al. 2010a) and Fermi/GBM (Paciesas 2010) at 21:06:19 UT. It had a duration of $T_{90}(15-350 \mathrm{keV})=0.3 \pm 0.05 \mathrm{~s}$ (Markwardt et al. 2010). Swift/XRT began observing the field $80 \mathrm{~s}$ after the BAT trigger and found a bright X-ray afterglow, which could be localized with an uncertainty of 4 .' 6 (radius) that could later be refined to 2.' 4 (Sbarufatti et al. 2010). UVOT started observing about $1 \mathrm{~min}$ later but could not find an optical counterpart (de Pasquale et al. 2010a,b). The optical afterglow was detected by Gemini-North $8.3 \mathrm{~h}$ after the burst with $r_{\mathrm{AB}}=25.46 \pm 0.20$ (Levan et al. 2010a). The burst is in detail discussed in Fong et al. (2011).

\section{C.10. GRB 100206A}

The burst triggered Swift/BAT at 13:30:05 UT (Krimm et al. 2010b) and had a duration of $T_{90}=0.12 \pm 0.03 \mathrm{~s}$ (Sakamoto et al. 2010). XRT started observing the field $75 \mathrm{~s}$ after the trigger and found an uncatalogued X-ray source (Krimm et al. 2010b), whose coordinates were later refined to RA, $\operatorname{Dec}(\mathbf{J} 2000)=$ 03:08:38.94, 13:09:25.5, with an error radius of 3'.2 (Goad et al. 2010a). The burst was also seen by Fermi/GBM with a spectral peak at $439_{-60}^{+73} \mathrm{keV}$, assuming a Band function (von Kienlin 2010). No optical counterpart was detected by Swift/UVOT (Krimm et al. 2010b; Marshall \& Krimm 2010) and other 
ground-based observatories (Bhattacharya et al. 2010; Guziy et al. 2010; Noda et al. 2010; Leloudas et al. 2010; Yurkov et al. 2010; Mao et al. 2010; Andreev et al. 2010; Rumyantsev et al. 2010). Evidence for a galaxy close to the XRT error circle was soon reported based on archival images of the field (Miller et al. 2010), whose redshift was later determined to be $z=0.41$ (Cenko et al. 2010a). Morgan et al. (2010) found that at this redshift this galaxy is very bright in $J H K$, suggesting that this is a luminous infrared galaxy. Levan et al. (2010c) speculated about the discovery of the faint optical afterglow of GRB 100206A based on William Herschel Telescope (WHT) observations starting $7 \mathrm{~h}$ after the burst. However, no fading of this source was seen on Gemini images taken 7 and $11.5 \mathrm{~h}$ after the event, suggesting that in fact this source could be the true GRB host galaxy (Berger et al. 2010b; Berger \& Chornock 2010). This placed a limit of $i>24.7$ on the brightness of the optical afterglow at $15.7 \mathrm{~h}$ after the burst (Berger \& Chornock 2010). The position of the XRT afterglow was slightly refined three years after the event (Evans 2011a,b). Perley et al. (2011) dispute that another faint source very close to the $z=0.41$ galaxy might instead be the host.

\section{C.11. GRB 100625A}

Swift/BAT triggered and located GRB 100625A at 18:32:28 UT (Holland et al. 2010a). The BAT light curve showed a single spike with a substructure and a duration of about $0.33 \mathrm{~s}$. XRT started observing the field $48 \mathrm{~s}$ after the trigger and found an uncatalogued X-ray source, whose coordinates were later refined to RA, Dec(J2000) = 01:03:10.98, -39:05:18.3, with an error radius of 1 '. 8 . No optical counterpart was detected by Swift/UVOT. The burst was also seen by Konus-Wind and Fermi. The Fermi/GBM light curve shows two closely spaced narrow pulses with a duration $\left(T_{90}\right)$ of about $0.32 \mathrm{~s}(50-300 \mathrm{keV}$; Holland et al. 2010b). Inside the XRT error circle, an object was reported in the optical bands by ground-based observatories (Levan \& Tanvir 2010; Berger et al. 2010a; Tanvir \& Levan 2010). However, the non-variation of the object and its extended shape pointed to it being a host galaxy candidate. The position of the XRT afterglow was slightly refined three years after the event (Evans 2011a,b).

\section{C.12. GRB $100628 A$}

Swift/BAT triggered and located GRB 100628A at 08:16:40 UT as did INTEGRAL (Beckmann et al. 2010). In the BAT window, it had a duration of $T_{90}(15-350 \mathrm{keV})=0.036 \pm 0.009 \mathrm{~s}$ (Immler et al. 2010). Swift/XRT began observing the field $86 \mathrm{~s}$ later and located an X-ray afterglow at coordinates RA, $\operatorname{Dec}(\mathrm{J} 2000)=15: 03: 52.95,-31: 39: 41.7$, with an error radius of 5'.2 (Starling \& Immler 2010). No optical afterglow was found, neither by UVOT nor by ground-based observatories
(Immler 2010; Burenin et al. 2010; Berger et al. 2010e,d; Suzuki et al. 2010; Levan et al. 2010b). Based on Magellan observations, Berger et al. (2010e) noticed, however, the presence of several galaxies close to and inside the XRT error circle (see also Berger 2010c). The position of the X-ray afterglow was later rejected when another faint X-ray source was found that had faded away. This source at coordinates RA, Dec $(\mathrm{J} 2000)=$ 15:03:52.41, $-31: 39: 30.2$ (error radius $7^{\prime \prime}$ ) is now considered as the most likely X-ray afterglow (Starling et al. 2010). Inside this error circle Berger (2010d) reports the presence of two galaxies; they, however, did not show any evidence of a superposed optical afterglow. For one of these galaxies, Cenko et al. (2010b) measured a redshift of $z=0.102$.

\section{C.13. GRB 100702A}

The burst triggered Swift/BAT at 01:03:47 UT (Siegel et al. 2010a). It was a FRED-like single-peaked burst with a duration of $T_{90}(15-350 \mathrm{keV})=0.16 \pm 0.03 \mathrm{~s}$ (Baumgartner et al. 2010). Swift slewed immediately to the burst and found a bright X-ray afterglow, which faded rapidly after an early plateau phase and was already undetected after the first orbit (Grupe \& Siegel 2010; Siegel et al. 2010b). No optical/NIR afterglow candidate was found, neither in rapid response observations by ROTSE-IIIc located at Mt. Gamsberg, Namibia (Flewelling et al. 2010) nor by optical observations with the acquisition camera of VLT/X-shooter (Malesani et al. 2010) and NIR observations using Persson's Auxilliary Nasmyth Infrared Camera (PANIC) at the Magellan/Baade telescope (Fong et al. 2010; Berger et al. 2010c).

\section{C.14. GRB 101219A}

GRB 101219A was a short-hard burst localized by Swift/BAT at 02:31:29 UT (Gelbord et al. 2010), which was also detected by Konus-Wind (Golenetskii et al. 2010). The BAT light curve consists of a single spike with a duration of $T_{90}(15-350 \mathrm{keV})=$ $0.6 \pm 0.2 \mathrm{~s}$ (Krimm et al. 2010a). A fading X-ray afterglow was found $60 \mathrm{~s}$ after the trigger, but no optical afterglow was detected (Gelbord et al. 2010). Inside the XRT error circle, a faint extended object was observed in the $i$ and $r$ band with the Gemini South telescope $43 \mathrm{~min}$ after the burst (Perley et al. 2010). In addition, the same faint source was detected in the $J$ band with the $6.5-\mathrm{m}$ Magellan Baade telescope $1.5 \mathrm{~h}$ after the trigger (Chornock et al. 2010). Second-epoch observations showed no variable source inside the XRT error circle. Spectroscopic observations performed on the host galaxy candidate with GMOS mounted at the Gemini-North telescope derived a redshift of $z=0.718$ (Chornock \& Berger 2011). The position of the XRT afterglow was slightly revised two years after the event (Evans 2011a,b). 